INSTITUT NATIONAL DE RECHERCHE EN INFORMATIQUE ET EN AUTOMATIQUE

\title{
A five equation reduced Model for compressible two phase flow problems.
}

\author{
Hervé Guillard — Angelo Murrone
}

$\mathbf{N}^{\circ} 4778$

Mars 2003

THÈME 4 



\title{
A five equation reduced Model for compressible two phase flow problems.
}

\author{
Hervé Guillard* , Angelo Murrone ${ }^{\dagger}$ \\ Thème 4 - Simulation et optimisation \\ de systèmes complexes \\ Projet SMASH \\ Rapport de recherche $\mathrm{n}^{\circ} 4778$ - Mars 2003 - 33 pages
}

\begin{abstract}
This paper presents an Euleriean model for the simulation of compressible two-phase flow problems. The starting point of the study is a seven equation, two pressure, two velocity model. This model contains relaxation terms that drive the systems toward pressure and velocity equilibrium. We perform an asymptotic analysis of this system in the limit of zero relaxation time and derive a five equation hyperbolic reduced system. We study the mathematical properties of the system, the structure of the waves and the expression of the Riemann's invariants. We then describe two different numerical approximation schemes for this system. The first one relies on a linearized Riemann solver while the second uses more heavily the mathematical structure of the system and relies on a linearization of the characteristic relations. Finally, we present some numerical experiments and comparison with the results obtained by the two pressure, two velocity model as well as some test cases in interface computations.
\end{abstract}

Key-words: Two phase flows, Compressible Interface problems, Hyperbolic relaxation, Riemann solver, Non conservative products.

* Herve.Guillard@sophia.inria.fr - Projet SMASH

$\dagger$ Angelo.Murrone@sophia.inria.fr - Projet SMASH et DTP/STH/LTA CEA Cadarache, 13108 Saint-Paul-Lez-Durance (France) 


\section{Un Modèle réduit à cinq équations pour les problèmes d'écoulements diphasiques compressibles.}

Résumé : Cet article présente un modèle Eulérien pour les problèmes d'écoulements diphasiques compressibles. Le point de départ de l'étude est un modèle à sept équations contenant deux pressions et deux vitesses. Ce modèle contient des termes sources de relaxation qui conduisent le système vers l'équilibre des pressions et des vitesses. Nous réalisons une analyse asymptotique de ce système dans la limite des temps de relaxation infiniment petits et dérivons un modèle réduit à cinq équations, inconditionellement hyperbolique. Nous étudions les propriétés mathématiques du système, la structure des ondes et l'expression des invariants de Riemann. Nous proposons ensuite deux schémas numériques pour ce système. Le premier est basé sur un solveur de Riemann linéarisé tandis que le deuxième utilise de manière plus forte la structure du modèle et se base sur la linéarisation des relations caractéristiques. Enfin nous présentons une série d'expériences numériques et des comparaisons avec les résultats donnés par le modèle à deux pressions et deux vitesses, ainsi que des expériences sur des problèmes d'interfaces.

Mots-clés : Ecoulements diphasiques, Problèmes d'interfaces entre fluides compressibles, Relaxation hyperbolique, Solveur de Riemann, Produits non conservatifs. 


\section{Acknowledgements}

The work of Angelo Murrone has been supported by a grant of the "Conseil Regional" of region PACA and the CEA Cadarache. Angelo Murrone wishes to acknowledge the support of M. Grandotto (CEA Cadarache), P. Gubernatis (CEA Cadarache) and R. Saurel (IUSTI Marseille). He would also like to thank his family for their constant support. We also address a warm thank to M.H. Lallemand for her help with the relaxation procedures in the numerical solution of the seven equation model. 


\section{Contents}

1 Introduction $\quad 6$

2 Derivation of the Model $\quad 7$

2.1 The seven equation model $\ldots \ldots \ldots \ldots \ldots \ldots \ldots \ldots \ldots$

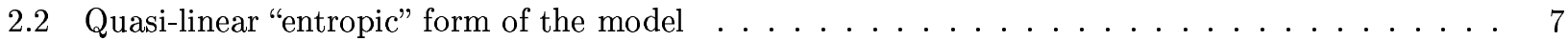

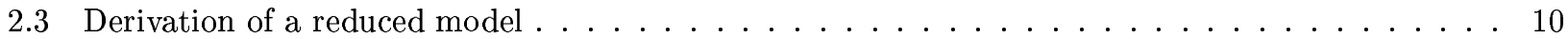

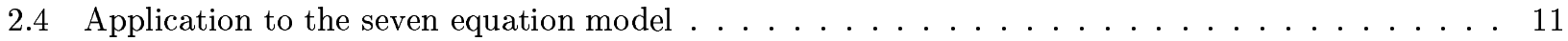

3 Mathematical Study $\quad 14$

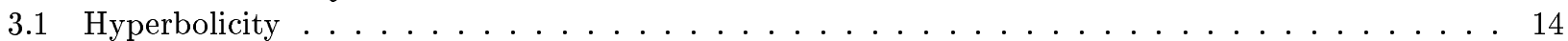

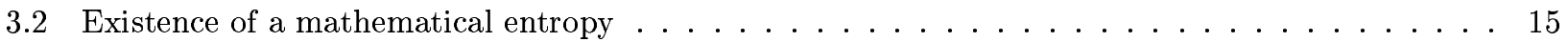

3.3 Structure of the waves $\ldots \ldots \ldots \ldots \ldots \ldots \ldots \ldots \ldots$

3.4 Riemann's invariants . . . . . . . . . . . . . . . . . . . . . . . 17

4 Numerical Approximation $\quad 18$

4.1 The VFRoe-ncv scheme . . . . . . . . . . . . . . . . . . . . . . . . . . 18

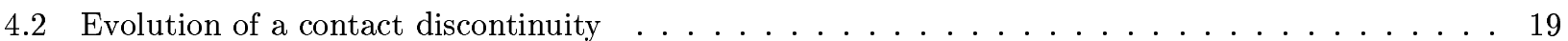

4.3 Acoustic Solver . . . . . . . . . . . . . . . . . . . . . . . 20

5 Numerical results $\quad 22$

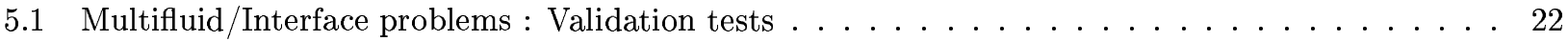

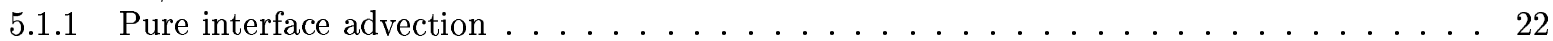

5.1 .2 Water-air shock tube . . . . . . . . . . . . . . . . . . 23

5.2 Two-phase flow problems : Comparison with the seven equation Model . . . . . . . . . . . 25

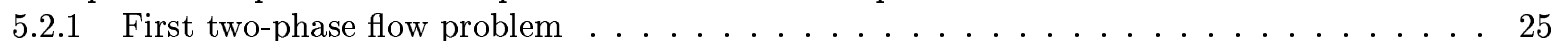

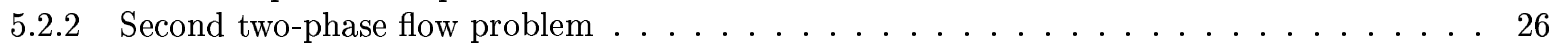

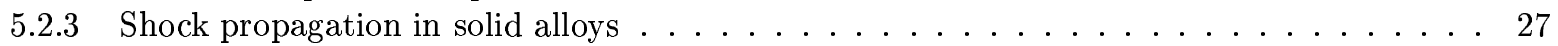

5.3 Two dimensional tests . . . . . . . . . . . . . . . . . . . . . . . . . 29

5.3.1 First two dimensional test : Bubble drop . . . . . . . . . . . . . . . . . 29

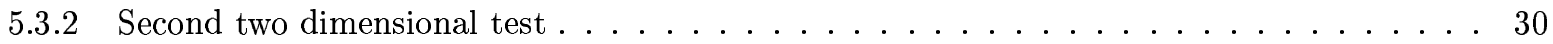

6 Conclusion $\quad 32$ 


\section{List of Figures}

1 Sound speed of an air-water mixture under atmospheric conditions for the 5 equation reduced model (solid line) and the 5 equation transport model (dotted line). . . . . 15

25 equation reduced model (left) and 5 equation transport model (right) in the pure interface advection : mixture variables. Computed solutions with 1000 cells (symbols). 22

35 equation reduced model (left) and 5 equation transport model (right) in the waterair shock tube : mixture variables. Computed solutions with 1000 cells (symbols) and exact solutions (dotted lines) . . . . . . . . . . . . . . . . 23

45 equation reduced model (left) and 5 equation transport model (right) in the waterair shock tube : phase variables. Computed solutions with 1000 cells (symbols). . . 24

55 equation reduced model (left) and 7 equation model (right) in the first two-phase flow problem. Computed solutions with 1000 cells (symbols) . . . . . . . . . . 25

65 equation reduced model (left) and 7 equation model (right) in the second two-phase flow problem. Computed solutions with 1000 cells (symbols) . . . . . . . . . 26

7 Schematic representation of the two-phase impact problem . . . . . . . . . . . 27

8 Results of the 5 equation reduced model for the epoxy/spinel under shock impact

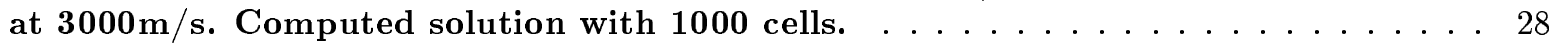

95 equation reduced model (solid line) and 7 equation model (dotted line) in the Mixture Hugoniot (epoxy/spinel) problem and experimental data (symbols) . . . . 28

10 Initial configuration for the first two dimensional test : bubble drop. . . . . . . . . . 29

11 Isovalues of the volume fraction for the first two dimensional test : bubble drop. . . 30

12 Initial configuration for the second two dimensional test. . . . . . . . . . . . 30

13 Isovalues of the volume fraction for the second two dimensional test. . . . . . . . . . 31 


\section{Introduction}

Modelling of two-phase flows is typically based on averaging procedures ([9], [7]). In their most general form, these averaging techniques produce models characterized by two differents velocities and pressures for each phase supplemented by one or several topological equations. Thus, in one dimension and for non-isentropic flows, a two-phase model of this type consists of at least 7 equations (two mass conservation equations, two momentum equations, two energy (or pressure) equations plus one topological equation). These type of models have been known for a long time [14], [16], [4] but have been seldom used due to their complexity. However, some recent works [1] have shown that they possess several advantages over the more classical 6 equation system : these models are unconditionally hyperbolic, they are able to treat multiphase mixtures as well as interface problems between pure fluids and they allow the treatment of fluids characterized by very different thermodynamics because each fluid uses its own equation of state. However these models are numerically complex to solve because of the large number of waves they contain and of the sensibility of the results with respect to the relaxation procedures. These facts motivate the research of cheaper models and the present work.

To reach this objective, we remark that in many cases, the typical time scales associated with the occurence of pressure and velocity equilibrium are small. This, a priori allows the construction of a reduced model based on an asymptotic analysis of the seven equation model in the limit of zero relaxation times. The procedure we use in this paper is therefore similar to the one proposed for detonation studies in [10].

The summary of this work is as follows. In section 2, we give a brief description of the seven equation model. Then we perform an asymptotic analysis of this model for zero relaxation times and obtain a limit system characterized in one dimension by five partial differential equations. In section 3, we study the mathematical properties of this reduced model ; in particular we show that it is an unconditional hyperbolic system whatever the state laws of the pure fluids are. We study its mathematical structure and prove that the system contains fields which are either genuinely non linear or linearly degenerate and finally, we give the expression of the Riemann's invariants. Then in section 4, we study two numerical procedures to solve this system. These numerical techniques do not require any hypothesis on the equation of state of each fluid. The first one of VFRoe-ncv type (see [5]) is based on the solution of a linearized Riemann's problem written in "entropic" variables. The second numerical scheme uses more heavily the mathematical structure of the reduced system and is an extension of the acoustic solver described for instance in [17] for the Euler system of gas dynamics. With respect to the different numerical test, the acoustic solver seems to be more robust than the VFRoe-ncv one when computing low Mach number two-phase flows. Finally in section 5, the model have been applied to several difficult physical problems. In each case, the method provides reliable results, is able to compute strong shock waves, and to deal with interface problems occuring in compressible multifluid flows. 


\section{Derivation of the Model}

\subsection{The seven equation model}

The starting point of this study is the seven equation model presented in [1] which is a slight variation of the Baer-Nunziato 1986 model [4]. In term of conservative variables ${ }^{t}\left(\alpha_{k} \rho_{k}, \alpha_{k} \rho_{k} \boldsymbol{u}_{k}, \alpha_{k} \rho_{k} e_{k}, \alpha_{2}\right)$, this model can be written :

$$
\begin{cases}\frac{\partial \alpha_{1} \rho_{1}}{\partial t}+\operatorname{div}\left(\alpha_{1} \rho_{1} \boldsymbol{u}_{1}\right) & =0 \\ \frac{\partial \alpha_{1} \rho_{1} \boldsymbol{u}_{1}}{\partial t}+\operatorname{div}\left(\alpha_{1} \rho_{1} \boldsymbol{u}_{1} \otimes \boldsymbol{u}_{1}\right)+\nabla \alpha_{1} p_{1} & =p_{\mathrm{I}} \nabla \alpha_{1}+\lambda\left(\boldsymbol{u}_{2}-\boldsymbol{u}_{1}\right) \\ \frac{\partial \alpha_{1} \rho_{1} e_{1}}{\partial t}+\operatorname{div}\left(\alpha_{1} \rho_{1} e_{1}+\alpha_{1} p_{1}\right) \boldsymbol{u}_{1} & =p_{\mathrm{I}} \frac{\partial \alpha_{2}}{\partial t}+\lambda \boldsymbol{u}_{\mathrm{I}} \cdot\left(\boldsymbol{u}_{2}-\boldsymbol{u}_{1}\right) \\ \frac{\partial \alpha_{2} \rho_{2}}{\partial t}+\operatorname{div}\left(\alpha_{2} \rho_{2} \boldsymbol{u}_{2}\right) & =0 \\ \frac{\partial \alpha_{2} \rho_{2} \boldsymbol{u}_{2}}{\partial t}+\operatorname{div}\left(\alpha_{2} \rho_{2} \boldsymbol{u}_{2} \otimes \boldsymbol{u}_{2}\right)+\nabla \alpha_{2} p_{2} & =p_{\mathrm{I}} \nabla \alpha_{2}-\lambda\left(\boldsymbol{u}_{2}-\boldsymbol{u}_{1}\right) \\ \frac{\partial \alpha_{2} \rho_{2} e_{2}}{\partial t}+\operatorname{div}\left(\alpha_{2} \rho_{2} e_{2}+\alpha_{2} p_{2}\right) \boldsymbol{u}_{2} & =-p_{\mathrm{I}} \frac{\partial \alpha_{2}}{\partial t}-\lambda \boldsymbol{u}_{\mathrm{I}} \cdot\left(\boldsymbol{u}_{2}-\boldsymbol{u}_{1}\right) \\ \frac{\partial \alpha_{2}}{\partial t}+\boldsymbol{u}_{\mathrm{I}} \cdot \nabla \alpha_{2} & =\mu\left(p_{2}-p_{1}\right)\end{cases}
$$

The notations are classical. $\alpha_{k}$ are the volume fractions of each phase $\left(\alpha_{1}+\alpha_{2}=1\right), \rho_{k}$ the phase densities, $\boldsymbol{u}_{k}$ the vector velocities, $p_{k}$ the pressures and $e_{k}=\varepsilon_{k}+\boldsymbol{u}_{k}^{2} / 2$ the specific total energies, with $\varepsilon_{k}$ the specific internal energies. On the other hand, $p_{\mathrm{I}}$ and $\boldsymbol{u}_{\mathrm{I}}$ stand for the interfacial pressure and velocity. In the Baer-Nunziato 1986 model [4], these variables are chosen as $p_{\mathrm{I}}=p_{2}$ and $\boldsymbol{u}_{\mathrm{I}}=\boldsymbol{u}_{1}$. But other choices are possible and for instance in [1], Saurel and Abgrall take the following interfacial values :

$$
\boldsymbol{u}_{\mathrm{I}}=\sum_{k=1}^{2} \alpha_{k} \rho_{k} \boldsymbol{u}_{k} / \sum_{k=1}^{2} \alpha_{k} \rho_{k} \quad \text { and } \quad p_{\mathrm{I}}=\sum_{k=1}^{2} \alpha_{k} p_{k}
$$

We note that the choice of interfacial velocity and pressure can have a deep impact on the structure of the waves present in this model and on the fulfilment of entropy inequalities (see [6]). However, in this paper as we will assume that the phase pressures and velocities relax to a common value, these choices are not important and will not affect the derivation of the reduced model. Actually, the model (1) contains relaxation parameters $\lambda$ and $\mu>0$ that determine the rates at which the velocities and pressures of the two-phases reach equilibrium. The rationale for the introduction of such terms is discussed for instance in [1]. Here we are interested in situations where the relaxation times are small compared with the others characteristic times of the flow. Thus we set $\lambda=\lambda^{\prime} / \varepsilon$ and $\mu=\mu^{\prime} / \varepsilon$ where $\lambda^{\prime}$ and $\mu^{\prime}$ are $\mathcal{O}(1)$ and we will analyse the case $\varepsilon \rightarrow 0$. This analysis can be performed directly on the system (1) with the conservative variables ${ }^{t}\left(\alpha_{k} \rho_{k}, \alpha_{k} \rho_{k} \boldsymbol{u}_{k}, \alpha_{k} \rho_{k} e_{k}, \alpha_{2}\right)$, however it is more convenient to work with the set of variables ${ }^{t}\left(s_{k}, \boldsymbol{u}_{k}, p_{k}, \alpha_{2}\right)$ where $s_{k}$ are the phase entropies and to use the quasi-linear form of the equations. This is the purpose of the next section.

\subsection{Quasi-linear "entropic" form of the model}

In the sequel, the material derivative of a quantity $\phi$ with respect to the velocities $\boldsymbol{u}_{k}$ of each phase $k$ as well as the interfacial velocity $\boldsymbol{u}_{\mathrm{I}}$ will be denoted by :

$$
\frac{D_{k} \phi}{D t}=\frac{\partial \phi}{\partial t}+\boldsymbol{u}_{k} \cdot \nabla \phi \quad \text { for } \quad k=1,2 \text { and } k=\mathrm{I}
$$


Using this notation, the momentum equations (1.2)-(1.5) and the mass conservation equations (1.1)-(1.4), it is easily seen that the velocities $\boldsymbol{u}_{k}$ obey the following equations :

$$
\begin{aligned}
& \alpha_{1} \rho_{1} \frac{D_{1} \boldsymbol{u}_{1}}{D t}+\nabla \alpha_{1} p_{1}=p_{\mathrm{I}} \nabla \alpha_{1}+\lambda\left(\boldsymbol{u}_{2}-\boldsymbol{u}_{1}\right) \\
& \alpha_{2} \rho_{2} \frac{D_{2} \boldsymbol{u}_{2}}{D t}+\nabla \alpha_{2} p_{2}=p_{\mathrm{I}} \nabla \alpha_{2}-\lambda\left(\boldsymbol{u}_{2}-\boldsymbol{u}_{1}\right)
\end{aligned}
$$

from which we deduce the equations for the kinetic energies $\boldsymbol{u}_{k}^{2} / 2$ of each phase :

$$
\begin{aligned}
& \alpha_{1} \rho_{1} \frac{D_{1} \boldsymbol{u}_{1}^{2} / 2}{D t}+\boldsymbol{u}_{1} . \nabla \alpha_{1} p_{1}=p_{\mathrm{I}} \boldsymbol{u}_{1} \cdot \nabla \alpha_{1}+\lambda \boldsymbol{u}_{1} \cdot\left(\boldsymbol{u}_{2}-\boldsymbol{u}_{1}\right) \\
& \alpha_{2} \rho_{2} \frac{D_{2} \boldsymbol{u}_{2}^{2} / 2}{D t}+\boldsymbol{u}_{2} . \nabla \alpha_{2} p_{2}=p_{\mathrm{I}} \boldsymbol{u}_{2} \cdot \nabla \alpha_{2}-\lambda \boldsymbol{u}_{2} \cdot\left(\boldsymbol{u}_{2}-\boldsymbol{u}_{1}\right)
\end{aligned}
$$

$>$ From (5.1)-(5.2), using $e_{k}=\varepsilon_{k}+\boldsymbol{u}_{k}^{2} / 2$ and the total energy equations (1.3)-(1.6), we get the equations for the specific internal energies $\varepsilon_{k}$ :

$$
\begin{aligned}
& \alpha_{1} \rho_{1} \frac{D_{1} \varepsilon_{1}}{D t}+\alpha_{1} p_{1} \operatorname{div} \boldsymbol{u}_{1}=p_{\mathrm{I}}\left(\boldsymbol{u}_{\mathrm{I}}-\boldsymbol{u}_{1}\right) \cdot \nabla \alpha_{1}+\mu p_{\mathrm{I}}\left(p_{2}-p_{1}\right)+\lambda\left(\boldsymbol{u}_{\mathrm{I}}-\boldsymbol{u}_{1}\right) \cdot\left(\boldsymbol{u}_{2}-\boldsymbol{u}_{1}\right) \\
& \alpha_{2} \rho_{2} \frac{D_{2} \varepsilon_{2}}{D t}+\alpha_{2} p_{2} \operatorname{div} \boldsymbol{u}_{2}=p_{\mathrm{I}}\left(\boldsymbol{u}_{\mathrm{I}}-\boldsymbol{u}_{2}\right) \cdot \nabla \alpha_{2}-\mu p_{\mathrm{I}}\left(p_{2}-p_{1}\right)-\lambda\left(\boldsymbol{u}_{\mathrm{I}}-\boldsymbol{u}_{2}\right) \cdot\left(\boldsymbol{u}_{2}-\boldsymbol{u}_{1}\right)
\end{aligned}
$$

Next, using the volume fraction equation (1.7), it is easily seen that we can rewrite the mass conservation equations (1.1)-(1.4) in term of phasic densities $\rho_{k}$ under the form :

$$
\begin{aligned}
\alpha_{1} \frac{D_{1} \rho_{1}}{D t}+\alpha_{1} \rho_{1} \operatorname{div} \boldsymbol{u}_{1} & =\rho_{1}\left(\boldsymbol{u}_{\mathrm{I}}-\boldsymbol{u}_{1}\right) \cdot \nabla \alpha_{1}+\mu \rho_{1}\left(p_{2}-p_{1}\right) \\
\alpha_{2} \frac{D_{2} \rho_{2}}{D t}+\alpha_{2} \rho_{2} \operatorname{div} \boldsymbol{u}_{2} & =\rho_{2}\left(\boldsymbol{u}_{\mathrm{I}}-\boldsymbol{u}_{2}\right) \cdot \nabla \alpha_{2}-\mu \rho_{2}\left(p_{2}-p_{1}\right)
\end{aligned}
$$

To obtain the equations for the phase entropies $s_{k}$, we use the Gibb's relation for each phase $k$ :

$$
d \varepsilon_{k}=T_{k} d s_{k}+\frac{p_{k}}{\rho_{k}^{2}} d \rho_{k} \quad \text { for } \quad k=1,2
$$

where $T_{k}$ is the temperature of phase $k$. Taking the material derivative of the Gibb's relation (8) and multiplying by $\alpha_{k} \rho_{k}$ we get :

$$
\alpha_{k} \rho_{k} T_{k} \frac{D_{k} s_{k}}{D t}=\alpha_{k} \rho_{k} \frac{D_{k} \varepsilon_{k}}{D t}-\frac{\alpha_{k} p_{k}}{\rho_{k}} \frac{D_{k} \rho_{k}}{D t} \quad \text { for } \quad k=1,2
$$

from which we deduce with (6.1)-(6.2)-(7.1)-(7.2) the equations for the phase entropies $s_{k}$ :

$$
\begin{aligned}
& \alpha_{1} \rho_{1} T_{1} \frac{D_{1} s_{1}}{D t}=\left(p_{\mathrm{I}}-p_{1}\right)\left(\boldsymbol{u}_{\mathrm{I}}-\boldsymbol{u}_{1}\right) \cdot \nabla \alpha_{1}+\mu\left(p_{\mathrm{I}}-p_{1}\right)\left(p_{2}-p_{1}\right)+\lambda\left(\boldsymbol{u}_{\mathrm{I}}-\boldsymbol{u}_{1}\right) \cdot\left(\boldsymbol{u}_{2}-\boldsymbol{u}_{1}\right) \\
& \alpha_{2} \rho_{2} T_{2} \frac{D_{2} s_{2}}{D t}=\left(p_{\mathrm{I}}-p_{2}\right)\left(\boldsymbol{u}_{\mathrm{I}}-\boldsymbol{u}_{2}\right) \cdot \nabla \alpha_{2}-\mu\left(p_{\mathrm{I}}-p_{2}\right)\left(p_{2}-p_{1}\right)-\lambda\left(\boldsymbol{u}_{\mathrm{I}}-\boldsymbol{u}_{2}\right) \cdot\left(\boldsymbol{u}_{2}-\boldsymbol{u}_{1}\right)
\end{aligned}
$$

Finally, to get the equations for the pressures $p_{k}$ of each phase, we write that $p_{k}=p_{k}\left(\rho_{k}, s_{k}\right)$ and obtain the following expression for the differential $d p_{k}$ :

$$
d p_{k}=\left(\frac{\partial p_{k}}{\partial \rho_{k}}\right)_{s_{k}} d \rho_{k}+\left(\frac{\partial p_{k}}{\partial s_{k}}\right)_{\rho_{k}} d s_{k} \quad \text { for } \quad k=1,2
$$

Now introducing the coefficients $\chi_{k}=\left(\partial \varepsilon_{k} / \partial \rho_{k}\right)_{p_{k}}$ and $\kappa_{k}=\left(\partial \varepsilon_{k} / \partial p_{k}\right)_{\rho_{k}}$, we write the differential of the specific internal energies $\varepsilon_{k}$ under the following form :

$$
d \varepsilon_{k}=\chi_{k} d \rho_{k}+\kappa_{k} d p_{k} \quad \text { for } \quad k=1,2
$$


Writing the equality of (8) and (12), solving for the differential of the pressure and comparing with (11) we get the well-known relations :

$$
a_{k}^{2}=\left(\frac{\partial p_{k}}{\partial \rho_{k}}\right)_{s_{k}}=\frac{1}{\kappa_{k}}\left(\frac{p_{k}}{\rho_{k}{ }^{2}}-\chi_{k}\right) \quad \text { and } \quad\left(\frac{\partial p_{k}}{\partial s_{k}}\right)_{\rho_{k}}=\frac{T_{k}}{\kappa_{k}} \quad \text { for } \quad k=1,2
$$

where $a_{k}$ denotes the sound speed of each phase. Now from (11)-(13) and after multiplication by the volume fraction $\alpha_{k}$, we obtain the relation :

$$
\alpha_{k} \frac{D_{k} p_{k}}{D t}=\frac{\alpha_{k}}{\kappa_{k}}\left(\frac{p_{k}}{\rho_{k}{ }^{2}}-\chi_{k}\right) \frac{D_{k} \rho_{k}}{D t}+\frac{\alpha_{k} T_{k}}{\kappa_{k}} \frac{D_{k} s_{k}}{D t} \quad \text { for } \quad k=1,2
$$

from which we deduce with (7.1)-(7.2)-(10.1)-(10.2) the equations for the pressures $p_{k}$ of each phase :

$$
\begin{aligned}
\alpha_{1} \frac{D_{1} p_{1}}{D t}+\alpha_{1} \rho_{1} a_{1}^{2} \operatorname{div} \boldsymbol{u}_{1} & =\frac{\rho_{1}}{\kappa_{1}}\left(\frac{p_{\mathrm{I}}}{\rho_{1}^{2}}-\chi_{1}\right)\left(\boldsymbol{u}_{\mathrm{I}}-\boldsymbol{u}_{1}\right) \cdot \nabla \alpha_{1}+\mu \frac{\rho_{1}}{\kappa_{1}}\left(\frac{p_{\mathrm{I}}}{\rho_{1}^{2}}-\chi_{1}\right)\left(p_{2}-p_{1}\right) \\
& +\frac{\lambda}{\kappa_{1} \rho_{1}}\left(\boldsymbol{u}_{\mathrm{I}}-\boldsymbol{u}_{1}\right) \cdot\left(\boldsymbol{u}_{2}-\boldsymbol{u}_{1}\right) \\
\alpha_{2} \frac{D_{2} p_{2}}{D t}+\alpha_{2} \rho_{2} a_{2}^{2} \operatorname{div} \boldsymbol{u}_{2} & =\frac{\rho_{2}}{\kappa_{2}}\left(\frac{p_{\mathrm{I}}}{\rho_{2}^{2}}-\chi_{2}\right)\left(\boldsymbol{u}_{\mathrm{I}}-\boldsymbol{u}_{2}\right) \cdot \nabla \alpha_{2}-\mu \frac{\rho_{2}}{\kappa_{2}}\left(\frac{p_{\mathrm{I}}}{\rho_{2}^{2}}-\chi_{2}\right)\left(p_{2}-p_{1}\right) \\
& -\frac{\lambda}{\kappa_{2} \rho_{2}}\left(\boldsymbol{u}_{\mathrm{I}}-\boldsymbol{u}_{2}\right) \cdot\left(\boldsymbol{u}_{2}-\boldsymbol{u}_{1}\right)
\end{aligned}
$$

Finally introducing the notation $a_{k \mathrm{I}}^{2}=\left(p_{\mathrm{I}} / \rho_{k}^{2}-\chi_{k}\right) / \kappa_{k}$ where $a_{k \mathrm{I}}$ stands for the sound speed of the phase $k$ at the interface, we can rewrite equations (15.1)-(15.2) under the form :

$$
\begin{aligned}
\alpha_{1} \frac{D_{1} p_{1}}{D t}+\alpha_{1} \rho_{1} a_{1}^{2} \operatorname{div} \boldsymbol{u}_{1} & =\rho_{1} a_{1 \mathrm{I}}^{2}\left(\boldsymbol{u}_{\mathrm{I}}-\boldsymbol{u}_{1}\right) \cdot \nabla \alpha_{1}+\mu \rho_{1} a_{1 \mathrm{I}}^{2}\left(p_{2}-p_{1}\right)+\frac{\lambda}{\kappa_{1} \rho_{1}}\left(\boldsymbol{u}_{\mathrm{I}}-\boldsymbol{u}_{1}\right) \cdot\left(\boldsymbol{u}_{2}-\boldsymbol{u}_{1}\right) \\
\alpha_{2} \frac{D_{2} p_{2}}{D t}+\alpha_{2} \rho_{2} a_{2}^{2} \operatorname{div} \boldsymbol{u}_{2} & =\rho_{2} a_{2 \mathrm{I}}^{2}\left(\boldsymbol{u}_{\mathrm{I}}-\boldsymbol{u}_{2}\right) \cdot \nabla \alpha_{2}-\mu \rho_{2} a_{2 \mathrm{I}}^{2}\left(p_{2}-p_{1}\right)-\frac{\lambda}{\kappa_{2} \rho_{2}}\left(\boldsymbol{u}_{\mathrm{I}}-\boldsymbol{u}_{2}\right) \cdot\left(\boldsymbol{u}_{2}-\boldsymbol{u}_{1}\right)
\end{aligned}
$$

Let us summarize the results of this section. In term of "entropic" variables ${ }^{t}\left(s_{k}, \boldsymbol{u}_{k}, p_{k}, \alpha_{2}\right)$, the seven equation model (1) can be written under the quasi-linear form :

$$
\begin{cases}\alpha_{1} \rho_{1} T_{1} \frac{D_{1} s_{1}}{D t} & =\left(p_{\mathrm{I}}-p_{1}\right)\left(\boldsymbol{u}_{\mathrm{I}}-\boldsymbol{u}_{1}\right) \cdot \nabla \alpha_{1}+\mu\left(p_{\mathrm{I}}-p_{1}\right)\left(p_{2}-p_{1}\right)+\lambda\left(\boldsymbol{u}_{\mathrm{I}}-\boldsymbol{u}_{1}\right) \cdot\left(\boldsymbol{u}_{2}-\boldsymbol{u}_{1}\right) \\ \alpha_{2} \rho_{2} T_{2} \frac{D_{2} s_{2}}{D t} & \left.=p_{\mathrm{I}}-p_{2}\right)\left(\boldsymbol{u}_{\mathrm{I}}-\boldsymbol{u}_{2}\right) \cdot \nabla \alpha_{2}-\mu\left(p_{\mathrm{I}}-p_{2}\right)\left(p_{2}-p_{1}\right)-\lambda\left(\boldsymbol{u}_{\mathrm{I}}-\boldsymbol{u}_{2}\right) \cdot\left(\boldsymbol{u}_{2}-\boldsymbol{u}_{1}\right) \\ \alpha_{1} \rho_{1} \frac{D_{1} \boldsymbol{u}_{1}}{D t}+\nabla \alpha_{1} p_{1} \nabla \alpha_{1}+\lambda\left(\boldsymbol{u}_{2}-\boldsymbol{u}_{1}\right) & \\ \alpha_{2} \rho_{2} \frac{D_{2} \boldsymbol{u}_{2}}{D t}+\nabla \alpha_{2} p_{2} & =p_{\mathrm{I}} \nabla \alpha_{2}-\lambda\left(\boldsymbol{u}_{2}-\boldsymbol{u}_{1}\right) \\ \alpha_{1} \frac{D_{1} p_{1}}{D t}+\alpha_{1} \rho_{1} a_{1}^{2} \operatorname{div} \boldsymbol{u}_{1} & =\rho_{1} a_{1 \mathrm{I}}^{2}\left(\boldsymbol{u}_{\mathrm{I}}-\boldsymbol{u}_{1}\right) \cdot \nabla \alpha_{1}+\mu \rho_{1} a_{1 \mathrm{I}}^{2}\left(p_{2}-p_{1}\right)+\frac{\lambda}{\kappa_{1} \rho_{1}}\left(\boldsymbol{u}_{\mathrm{I}}-\boldsymbol{u}_{1}\right) \cdot\left(\boldsymbol{u}_{2}-\boldsymbol{u}_{1}\right) \\ \alpha_{2} \frac{D_{2} p_{2}}{D t}+\alpha_{2} \rho_{2} a_{2}^{2} \operatorname{div} \boldsymbol{u}_{2} & =\rho_{2} a_{2 \mathrm{I}}^{2}\left(\boldsymbol{u}_{\mathrm{I}}-\boldsymbol{u}_{2}\right) \cdot \nabla \alpha_{2}-\mu \rho_{2} a_{2 \mathrm{I}}^{2}\left(p_{2}-p_{1}\right)-\frac{\lambda}{\kappa_{2} \rho_{2}}\left(\boldsymbol{u}_{\mathrm{I}}-\boldsymbol{u}_{2}\right) \cdot\left(\boldsymbol{u}_{2}-\boldsymbol{u}_{1}\right) \\ \frac{D_{\mathrm{I}} \alpha_{2}}{D t} & =\mu\left(p_{2}-p_{1}\right)\end{cases}
$$




\subsection{Derivation of a reduced model}

In this section, to simplify the notation, we present only the one dimensional case or alternatively the expression $A(\boldsymbol{U}) \partial \boldsymbol{U} / \partial x$ can be understood as a shorthand notation for $\sum_{j=1}^{d} A_{j}(\boldsymbol{U}) \partial \boldsymbol{U} / \partial x_{j}$.

The derivation of the model can be presented briefly as follows : Consider an hyperbolic system with stiff source term :

$$
\frac{\partial \boldsymbol{U}}{\partial t}+A(\boldsymbol{U}) \frac{\partial \boldsymbol{U}}{\partial x}=\frac{R(\boldsymbol{U})}{\varepsilon}
$$

In this equation $\boldsymbol{U}=\boldsymbol{U}(x, t)$ the state vector belongs to $\Omega$, some open subset of $\mathbb{R}^{N}$. We assume that (18) is hyperbolic i.e for any $\boldsymbol{U} \in \Omega$, the matrix $A(\boldsymbol{U})$ is diagonalizable in $\mathbb{R}$ and possesses a complete set of eigenvectors.

We are interested in the behavior of the solutions of (18) when the relaxation time $\varepsilon$ goes to zero. Therefore, we expect these solutions to be close to $\mathcal{E}$ the subset of $\mathbb{R}^{N}$ defined by :

$$
\mathcal{E}=\left\{\boldsymbol{U} \in \mathbb{R}^{N} ; R(\boldsymbol{U})=0\right\}
$$

We make the following assumption :

Assumption 1 : The set of equations $R(\boldsymbol{U})=0$ defines a smooth manifold of dimension $n$. Moreover, for any $\boldsymbol{U} \in \mathcal{E}$ we explicitly know a parametrization $M$ from $\omega$ an open subset of $\mathbb{R}^{n}$ on $V$ a neighborhood of $\boldsymbol{U}$ in $\mathcal{E}$.

We call $\mathcal{E}$ the equilibrium manifold while in reference to the Boltzmann equation, the smooth diffeomorphism $M$ will be called the "Maxwellian". We note that for any $\boldsymbol{u} \in \omega$ the Jacobian matrix $d M_{\boldsymbol{u}}$ is a full rank matrix. Moreover we have the following result :

Proposition 1 : The column vectors of $d M \boldsymbol{u}$ form a basis of ker $R^{\prime}(M(\boldsymbol{u}))$.

Proof: Denote $T_{\boldsymbol{U}}(\mathcal{E})$ the tangent space of $\mathcal{E}$ at $\boldsymbol{U}$. Since $M$ is a diffeomorphism from $\omega$ onto $V \subset \mathcal{E}, d M_{\boldsymbol{u}}$ is a bijection from $\mathbb{R}^{n}$ onto $T_{M(\boldsymbol{u})}(\mathcal{E})$. Therefore the column vectors of $d M \boldsymbol{u}$ are $n$ independent vectors that form a basis of $T_{M(\boldsymbol{u})}(\mathcal{E})$. But, since the equilibrium manifold $\mathcal{E}$ is defined by the implicit equations (19), the result simply follows by noting that $T_{M(\boldsymbol{u})}(\mathcal{E})$ is precisely $\operatorname{ker} R^{\prime}(M(\boldsymbol{u}))$.

We will denote the column vectors of $d M_{\boldsymbol{u}},\left\{d M_{\boldsymbol{u}}^{1}, \ldots, d M_{\boldsymbol{u}}^{n}\right\}$. Now let $\left\{I^{1}, \ldots, I^{N-n}\right\}$ be a basis of $\operatorname{Rng}\left(R^{\prime}(M(\boldsymbol{u}))\right)$ the range of $R^{\prime}(M(\boldsymbol{u}))$ and define the $N \times N$ matrix $S$ by :

$$
S=\left[d M_{\boldsymbol{u}}^{1}, \ldots, d M_{\boldsymbol{u}}^{n}, I^{1}, \ldots, I^{N-n}\right]
$$

Thanks to proposition $1, S$ is an invertible matrix. Let us denote by $P$ and $Q$ the $n \times N$ and $N-n \times N$ matrices composed of the first $n$ and last $N-n$ rows respectively of its inverse, we have :

Proposition 2 : Let $\operatorname{ker}\left(R^{\prime}(M(\boldsymbol{u}))\right)$ and $\operatorname{Rng}\left(R^{\prime}(M(\boldsymbol{u}))\right)$ be equipped respectively with the basis $\left\{d M_{\boldsymbol{u}}^{1}, \ldots, d M_{\boldsymbol{u}}^{n}\right\}$ and $\left\{I^{1}, \ldots, I^{N-n}\right\}$, then in these basis $P$ and $Q$ are respectively the projection on $\operatorname{ker}\left(R^{\prime}(M(u))\right)$ in the direction of $\operatorname{Rng}\left(R^{\prime}(M(\boldsymbol{u}))\right)$ and the projection on $\operatorname{Rng}\left(R^{\prime}(M(\boldsymbol{u}))\right)$ in the direction of $\operatorname{ker}\left(R^{\prime}(M(\boldsymbol{u}))\right)$.

Proof : Let $\boldsymbol{v} \in \mathbb{R}^{n}, \boldsymbol{w} \in \mathbb{R}^{N-n}$ and for $\boldsymbol{U} \in \mathbb{R}^{N}$ let us write :

$$
\boldsymbol{U}=\sum_{j=1}^{n} \boldsymbol{v}^{j} d M_{\boldsymbol{u}}^{j}+\sum_{j=1}^{N-n} \boldsymbol{w}^{j} I^{j}
$$

then the vectors $\sum_{j=1}^{n} \boldsymbol{v}^{j} d M_{\boldsymbol{u}}^{j}$ and $\sum_{j=1}^{N-n} \boldsymbol{w}^{j} I^{j}$ are clearly respectively the projection on $\operatorname{ker}\left(R^{\prime}(M(\boldsymbol{u}))\right)$ in the direction of $\operatorname{Rng}\left(R^{\prime}(M(u))\right)$ of $\boldsymbol{U}$ and the projection on $\operatorname{Rng}\left(R^{\prime}(M(\boldsymbol{u}))\right)$ in the direction of $\operatorname{ker}\left(R^{\prime}(M(\boldsymbol{u}))\right)$ of $\boldsymbol{U}$. In matrix form (21) can be written :

$$
U=S\left[\begin{array}{c}
v \\
w
\end{array}\right]
$$

from which we deduce :

$$
\left[\begin{array}{c}
\boldsymbol{v} \\
\boldsymbol{w}
\end{array}\right]=S^{-1} \boldsymbol{U}=\left[\begin{array}{l}
P \boldsymbol{U} \\
Q \boldsymbol{U}
\end{array}\right]
$$


We note the following simple properties :

Proposition 3 : We have

1. P.dMu $=\operatorname{Id}(n)$ where $\operatorname{Id}(n)$ is the $n$ by $n$ identity matrix

2. $P \cdot R^{\prime}(M(\boldsymbol{u}))=0$

Proof: The first result follows from $S^{-1} . S=I d(N)$. To prove the second result, we note that for any $\boldsymbol{U} \in \mathbb{R}^{n}$, obviously $R^{\prime}(M(\boldsymbol{u})) \boldsymbol{U}$ is in the range of $R^{\prime}(M(\boldsymbol{u}))$.

Note that Assumption 1 is a very weak one. However, we emphazise that this assumption gives only the possibility to write a formal asymptotic expansion. In particular, this weak assumption does not imply that the reduced system is well-posed or hyperbolic nor does it implies that the expansion is convergent. More stronger assumptions are needed to establish results of this type. For a survey on some recent results on hyperbolic systems with stiff relaxation terms, the interested reader can see [13].

Now, to obtain a reduced model, we look for a solution in the form :

$$
\boldsymbol{U}=M(\boldsymbol{u})+\varepsilon \boldsymbol{V}
$$

Introducing this expression in (18) gives :

$$
\begin{aligned}
& \frac{\partial M(\boldsymbol{u})}{\partial t}+A(M(\boldsymbol{u})) \frac{\partial M(\boldsymbol{u})}{\partial x}-R^{\prime}(M(\boldsymbol{u})) \cdot \boldsymbol{V} \\
& +\varepsilon\left[\frac{\partial \boldsymbol{V}}{\partial t}+A(M(\boldsymbol{u})) \frac{\partial \boldsymbol{V}}{\partial x}+\left[\frac{\partial A}{\partial \boldsymbol{U}_{i}} \boldsymbol{V}_{i}\right] \frac{\partial M(\boldsymbol{u})}{\partial x}-\frac{1}{2} R^{\prime \prime}(M(\boldsymbol{u}))(\boldsymbol{V}, \boldsymbol{V})\right]=\mathcal{O}\left(\varepsilon^{2}\right)
\end{aligned}
$$

Thanks to proposition 3, multiplying this equation by $P$ gives :

$$
\frac{\partial \boldsymbol{u}}{\partial t}+P \cdot A(M(\boldsymbol{u})) \cdot d M u \frac{\partial \boldsymbol{u}}{\partial x}=\mathcal{O}(\varepsilon)
$$

The reduced model of (18) is thus obtained by neglecting the terms of order $\varepsilon$.

\subsection{Application to the seven equation model}

The application of the previous asymptotic analysis to the seven equation model (17) is straighforward. We just need to identify the Maxwellian $M(\boldsymbol{u})$ and then to compute the Jacobian matrix $d M_{\boldsymbol{u}}$ and the projection matrix $P$. Let $\boldsymbol{u}={ }^{t}\left(s_{1}, s_{2}, u, p, \alpha_{2}\right) \in \mathbb{R}^{5}$ the Maxwellian is defined by :

$$
\boldsymbol{u} \rightarrow \boldsymbol{U}=M(\boldsymbol{u})=\left(\begin{array}{c}
s_{1} \\
s_{2} \\
u \\
u \\
p \\
p \\
\alpha_{2}
\end{array}\right)
$$

The jacobian matrix of this transformation is :

$$
d M_{\boldsymbol{u}}=\left(\begin{array}{ccccc}
1 & 0 & 0 & 0 & 0 \\
0 & 1 & 0 & 0 & 0 \\
0 & 0 & 1 & 0 & 0 \\
0 & 0 & 1 & 0 & 0 \\
0 & 0 & 0 & 1 & 0 \\
0 & 0 & 0 & 1 & 0 \\
0 & 0 & 0 & 0 & 1
\end{array}\right)
$$


The linearized source term evaluated on a Maxwellian is :

$$
R^{\prime}(M(\boldsymbol{u}))=\left(\begin{array}{ccccccc}
0 & 0 & 0 & 0 & 0 & 0 & 0 \\
0 & 0 & 0 & 0 & 0 & 0 & 0 \\
0 & 0 & -1 & 1 & 0 & 0 & 0 \\
0 & 0 & 1 & -1 & 0 & 0 & 0 \\
0 & 0 & 0 & 0 & -1 & 1 & 0 \\
0 & 0 & 0 & 0 & 1 & -1 & 0 \\
0 & 0 & 0 & 0 & -1 & 1 & 0
\end{array}\right)
$$

A basis of the range $\operatorname{Rng}\left(R^{\prime}(M(u))\right)$ of this matrix is the set :

$$
I^{1}=\left(\begin{array}{c}
0 \\
0 \\
-1 \\
1 \\
0 \\
0 \\
0
\end{array}\right) ; \quad I^{2}=\left(\begin{array}{c}
0 \\
0 \\
0 \\
0 \\
-1 \\
1 \\
-1
\end{array}\right)
$$

The inversion of the matrix $S=\left[d M_{\boldsymbol{u}}^{1}, \ldots, d M_{\boldsymbol{u}}^{5}, I^{1}, I^{2}\right]$ thus gives the projection on $\operatorname{ker}\left(R^{\prime}(M(\boldsymbol{u}))\right)$ :

$$
P=\left(\begin{array}{ccccccc}
1 & 0 & 0 & 0 & 0 & 0 & 0 \\
0 & 1 & 0 & 0 & 0 & 0 & 0 \\
0 & 0 & 1 / 2 & 1 / 2 & 0 & 0 & 0 \\
0 & 0 & 0 & 0 & 1 / 2 & 1 / 2 & 0 \\
0 & 0 & 0 & 0 & -1 / 2 & 1 / 2 & 1
\end{array}\right)
$$

To obtain, the reduced model, we just need now to compute the matrix product $P \cdot A(M(\boldsymbol{u})) . d M \boldsymbol{u}$. After some straighforward computations, we then get a five equation reduced model written in term of "entropic" variables ${ }^{t}\left(s_{1}, s_{2}, \boldsymbol{u}, p, \alpha_{2}\right)$ :

$$
\begin{cases}\frac{D s_{1}}{D t} & =0 \\ \frac{D s_{2}}{D t} & =0 \\ \frac{D u}{D t}+\frac{1}{\rho} \nabla p & =0 \\ \frac{D p}{D t}+\rho \hat{a}^{2} \operatorname{div} u & =0 \\ \frac{D \alpha_{2}}{D t} & =\alpha_{1} \alpha_{2} \frac{\rho_{1} a_{1}^{2}-\rho_{2} a_{2}^{2}}{\sum_{k=1}^{2} \alpha_{k^{\prime}} \rho_{k} a_{k}^{2}} \operatorname{div} \boldsymbol{u}\end{cases}
$$

where we have introduced the notation $D \phi / D t=\partial \phi / \partial t+\boldsymbol{u} . \nabla \phi$, the mixture density $\rho$ :

$$
\rho=\sum_{k=1}^{2} \alpha_{k} \rho_{k}
$$

and also the averaged sound speed $\hat{a}$ defined by the following formula :

$$
\frac{1}{\rho \hat{a}^{2}}=\sum_{k=1}^{2} \frac{\alpha_{k}}{\rho_{k} a_{k}^{2}}
$$


To end this section, we note that after some algebraic manipulations, the model (27) may be written in term of conservative variables ${ }^{t}\left(\alpha_{1} \rho_{1}, \alpha_{2} \rho_{2}, \rho \boldsymbol{u}, \rho e, \alpha_{2}\right)$ as :

$$
\begin{cases}\frac{\partial \alpha_{1} \rho_{1}}{\partial t}+\operatorname{div}\left(\alpha_{1} \rho_{1} \boldsymbol{u}\right) & =0 \\ \frac{\partial \alpha_{2} \rho_{2}}{\partial t}+\operatorname{div}\left(\alpha_{2} \rho_{2} \boldsymbol{u}\right) & =0 \\ \frac{\partial \rho \boldsymbol{u}}{\partial t}+\operatorname{div}(\rho \boldsymbol{u} \otimes \boldsymbol{u})+\nabla p & =0 \\ \frac{\partial \rho e}{\partial t}+\operatorname{div}(\rho e+p) \boldsymbol{u} & =0 \\ \frac{\partial \alpha_{2}}{\partial t}+\boldsymbol{u} . \nabla \alpha_{2} & =\alpha_{1} \alpha_{2} \frac{\rho_{1} a_{1}^{2}-\rho_{2} a_{2}^{2}}{\sum_{k=1}^{2} \alpha_{k^{\prime}} \rho_{k} a_{k}^{2}} \operatorname{div} \boldsymbol{u}\end{cases}
$$

where $e$ the specific total energy is defined by $e=\varepsilon+\boldsymbol{u}^{2} / 2$ while the specific internal energy $\varepsilon$ is given by the relation $\rho \varepsilon=\sum_{k=1}^{2} \alpha_{k} \rho_{k} \varepsilon_{k}\left(p, \rho_{k}\right)$.

Remark : In [12] and [3], a diffuse interface method for the simulation of interfaces between compressible fluids was introduced. This model (in the sequel referred to as the "five equation-transport model") is very similar to (30) except that instead of (30.5), equation for the volume fraction is simply a transport equation :

$$
\frac{\partial \alpha_{2}}{\partial t}+\boldsymbol{u} \cdot \nabla \alpha_{2}=0
$$

In view of (30.5) and since for interface problems, the zone where the product $\alpha_{1} \alpha_{2}$ is expected to be small, the differences between the two models seem unimportant. However, as we will see the mathematical properties of the two models are quite different. In particular, the "five equation-transport model" of [12] and [3] is not compatible with the fact that the material derivatives of the phase entropies are zero. This is quite obvious from the form (27) of the present reduced model. But it can also be seen as follows : Let us write the equality of the pressures in the two phases :

$$
p_{1}\left(s_{1}, \rho_{1}\right)=p_{2}\left(s_{2}, \rho_{2}\right)
$$

and take the material derivative of this expression to obtain :

$$
\left(\frac{\partial p_{1}}{\partial s_{1}}\right)_{\rho_{1}} \frac{D s_{1}}{D t}-\left(\frac{\partial p_{2}}{\partial s_{2}}\right)_{\rho_{2}} \frac{D s_{2}}{D t}=\left(\frac{\partial p_{2}}{\partial \rho_{2}}\right)_{s_{2}} \frac{D \rho_{2}}{D t}-\left(\frac{\partial p_{1}}{\partial \rho_{1}}\right)_{s_{1}} \frac{D \rho_{1}}{D t}
$$

Now, with (30.1)-(30.2) we have :

$$
\frac{D \rho_{k}}{D t}=\frac{1}{\alpha_{k}}\left[\frac{D \alpha_{k} \rho_{k}}{D t}-\rho_{k} \frac{D \alpha_{k}}{D t}\right]=-\frac{1}{\alpha_{k}}\left[\alpha_{k} \rho_{k} \operatorname{div} \boldsymbol{u}+\rho_{k} \frac{D \alpha_{k}}{D t}\right]
$$

and we get from (33) :

$$
\left(\frac{\partial p_{1}}{\partial s_{1}}\right)_{\rho_{1}} \frac{D s_{1}}{D t}-\left(\frac{\partial p_{2}}{\partial s_{2}}\right)_{\rho_{2}} \frac{D s_{2}}{D t}+\left(\rho_{1} a_{1}^{2}-\rho_{2} a_{2}^{2}\right) \operatorname{div} \boldsymbol{u}=\left(\frac{\rho_{2} a_{2}^{2}}{\alpha_{2}}+\frac{\rho_{1} a_{1}^{2}}{\alpha_{1}}\right) \frac{D \alpha_{2}}{D t}
$$

that shows that equation (30.5) is a direct consequence of the assumptions $D s_{1} / D t=D s_{2} / D t=0$ and conversely that the assumptions $D \alpha_{2} / D t=0$ is not compatible with these relations. Actually, (35) shows that if $D \alpha_{2} / D t=0$ then the phase entropies must evolve according to the relation :

$$
\left(\frac{\partial p_{1}}{\partial s_{1}}\right)_{\rho_{1}} \frac{D s_{1}}{D t}-\left(\frac{\partial p_{2}}{\partial s_{2}}\right)_{\rho_{2}} \frac{D s_{2}}{D t}=\left(\rho_{2} a_{2}^{2}-\rho_{1} a_{1}^{2}\right) \operatorname{div} \boldsymbol{u}
$$




\section{Mathematical Study}

For regular solutions, the mathematical study of the model can be performed with any set of independant variables. Here, for instance, we will use the set of variables ${ }^{t}\left(s_{1}, s_{2}, \boldsymbol{u}, p, Y_{2}\right)$ where $Y_{k}=\alpha_{k} \rho_{k} / \rho$ stand for the mass fractions of each phase. It is easily seen from the mass conservation equations (30.1)-(30.2) that $Y_{k}$ obey the equations $D Y_{k} / D t=0$ and the five equation reduced model can be written :

$$
\begin{cases}\frac{D s_{1}}{D t} & =0 \\ \frac{D s_{2}}{D t} & =0 \\ \frac{D \boldsymbol{u}}{D t}+\frac{1}{\rho} \nabla p & =0 \\ \frac{D p}{D t}+\rho \hat{a}^{2} \operatorname{div} \boldsymbol{u} & =0 \\ \frac{D Y_{2}}{D t} & =0\end{cases}
$$

where the mixture density $\rho$ and the averaged sound speed $\hat{a}$ have been defined in the previous section 2 . On the other hand, the system is clearly invariant by rotation. As a consequence for the sake of simplicity, we can perform the mathematical study for the system written in one dimension.

\subsection{Hyperbolicity}

So if we set $\boldsymbol{q}={ }^{t}\left(s_{1}, s_{2}, u, p, Y_{2}\right)$, for smooth solutions, the system (37) can be written in one dimension as :

$$
\frac{\partial \boldsymbol{q}}{\partial t}+A(\boldsymbol{q}) \frac{\partial \boldsymbol{q}}{\partial x}=0
$$

where the matrix $A(\boldsymbol{q})$ is defined by :

$$
A(\boldsymbol{q})=\left(\begin{array}{ccccc}
u & 0 & 0 & 0 & 0 \\
0 & u & 0 & 0 & 0 \\
0 & 0 & u & 1 / \rho & 0 \\
0 & 0 & \rho \hat{a}^{2} & u & 0 \\
0 & 0 & 0 & 0 & u
\end{array}\right)
$$

Since the characteristic equation of the matrix $A(\boldsymbol{q})$ is given by $(u-\lambda)^{3}\left((u-\lambda)^{2}-\hat{a}^{2}\right)=0$, we obtain three distinct eigenvalues for the matrix $A(\boldsymbol{q})$ :

$$
\left\{\begin{array}{l}
\lambda_{1}(\boldsymbol{q})=u-\hat{a} \\
\lambda_{2}(\boldsymbol{q})=\lambda_{3}(\boldsymbol{q})=\lambda_{4}(\boldsymbol{q})=u \\
\lambda_{5}(\boldsymbol{q})=u+\hat{a}
\end{array}\right.
$$

where $\hat{a}$ is given by the relation (29). Thus $\hat{a}$ is clearly real and all the eigenvalues of the matrix $A(\boldsymbol{q})$ are real. The expression (29) where the average "acoustic impedance" $\rho \hat{a}^{2}$ appears as the harmonic average of the "acoustic impedances" of the pure phases $\rho_{k} a_{k}^{2}$ implies that the sound speed of a mixture can be less than the sound speed of either phase. This fact is well-known in the two-phase litterature (see for instance [16] or [7]).

The right eigenvectors $r_{i}(\boldsymbol{q})$ (for $i \in\{1, \ldots, 5\}$ ) which verify the relation $A(\boldsymbol{q}) r_{i}(\boldsymbol{q})=\lambda_{i}(\boldsymbol{q}) r_{i}(\boldsymbol{q})$ can be choosen as :

$$
r_{1}(\boldsymbol{q})=\left(\begin{array}{c}
0 \\
0 \\
\hat{a} \\
-\rho \hat{a}^{2} \\
0
\end{array}\right) r_{2}(\boldsymbol{q})=\left(\begin{array}{l}
1 \\
0 \\
0 \\
0 \\
0
\end{array}\right) \quad r_{3}(\boldsymbol{q})=\left(\begin{array}{l}
0 \\
1 \\
0 \\
0 \\
0
\end{array}\right) r_{4}(\boldsymbol{q})=\left(\begin{array}{l}
0 \\
0 \\
0 \\
0 \\
1
\end{array}\right) r_{5}(\boldsymbol{q})=\left(\begin{array}{c}
0 \\
0 \\
\hat{a} \\
\rho \hat{a}^{2} \\
0
\end{array}\right)
$$


We denote also by $l_{i}(\boldsymbol{q})$ (for $i \in\{1, \ldots, 5\}$ ) the left eigenvectors which obey the relation ${ }^{t} A(\boldsymbol{q}) l_{i}(\boldsymbol{q})=\lambda_{i}(\boldsymbol{q}) l_{i}(\boldsymbol{q})$. After normalization of the left and right eigenvectors to have ${ }^{t} l_{i}(\boldsymbol{q}) \cdot r_{j}(\boldsymbol{q})=\delta_{i j}$, we get :

$$
l_{1}(\boldsymbol{q})=\left(\begin{array}{c}
0 \\
0 \\
1 / 2 \hat{a} \\
-1 / 2 \rho \hat{a}^{2} \\
0
\end{array}\right) \quad l_{2}(\boldsymbol{q})=\left(\begin{array}{c}
1 \\
0 \\
0 \\
0 \\
0
\end{array}\right) \quad l_{3}(\boldsymbol{q})=\left(\begin{array}{c}
0 \\
1 \\
0 \\
0 \\
0
\end{array}\right) \quad l_{4}(\boldsymbol{q})=\left(\begin{array}{l}
0 \\
0 \\
0 \\
0 \\
1
\end{array}\right) \quad l_{5}(\boldsymbol{q})=\left(\begin{array}{c}
0 \\
0 \\
1 / 2 \hat{a} \\
1 / 2 \rho \hat{a}^{2} \\
0
\end{array}\right)
$$

The system is thus clearly hyberbolic since the matrix $A(\boldsymbol{q})$ is diagonalizable in $\mathbb{R}$ and its eigenvectors span the whole space $\mathbb{R}^{5}$. We note that this system is unconditionally hyperbolic as soon as the sound speed in the pure fluids are real whatever the state laws of the pure fluids are.

Remark : In the "five equation-transport model" of [12], [3], the sound speed is given by the expression :

$$
\xi \hat{a}^{2}=\sum_{k=1}^{2} \xi_{k} Y_{k} a_{k}^{2}
$$

where $\xi_{k}=\left(\partial \rho_{k} \varepsilon_{k} / \partial p_{k}\right)_{\rho_{k}}$ and $\xi=\sum_{k=1}^{2} \alpha_{k} \xi_{k}$. Thus as in (29), the sound speed of the mixture appears as some average of the phase sound speeds. However, these averages are different in the two models. In some extreme cases, for real fluids the coefficients $\xi_{k}$ can be negative and the formula (43) does not guarantee that the mixture speed of sound is real while formula (29) will always yield a real speed of sound.

Figure 1 displays the sound speed of an air-water mixture with respect to the air volume fraction, under atmospheric conditions, respectively for the five equation reduced model and the "five equation-transport model". One can notice that the values of the sound speeds are very different in the two models.

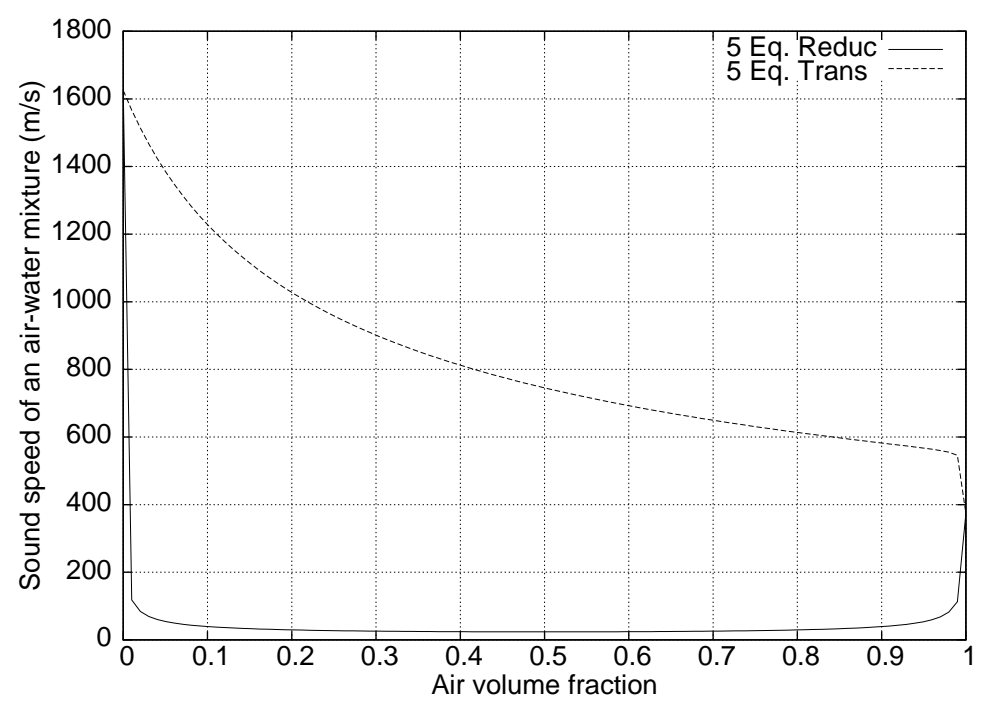

Figure 1: Sound speed of an air-water mixture under atmospheric conditions for the 5 equation reduced model (solid line) and the 5 equation transport model (dotted line).

\subsection{Existence of a mathematical entropy}

Since the phase entropies $s_{1}, s_{2}$ satisfy (37.1)-(37.2), the existence of an entropy for the five equation reduced system is obvious. The simplest choice is to define $S$ by :

$$
\rho S=\alpha_{1} \rho_{1} s_{1}+\alpha_{2} \rho_{2} s_{2}=\rho Y_{1} s_{1}+\rho Y_{2} s_{2}
$$

and we have :

$$
\frac{\partial \rho S}{\partial t}+\operatorname{div}(\rho S \boldsymbol{u})=0
$$




\subsection{Structure of the waves}

In this section, we analyze the structure of the waves. The objective is to show that the system contains fields which are either genuinely non linear or linearly degenerate (see for instance [8]). So let us start with the characteristic fields associated to the waves $u-\hat{a}$ and $u+\hat{a}$.

Proposition 4 : The characteristic fields associated to the waves $\lambda_{1}(\boldsymbol{q})=u-\hat{a}$ and $\lambda_{5}(\boldsymbol{q})=u+\hat{a}$ are genuinely non linear i.e we have $\nabla \boldsymbol{q} \lambda_{1}(\boldsymbol{q}) . r_{1}(\boldsymbol{q}) \neq 0$ and $\nabla \boldsymbol{q} \lambda_{5}(\boldsymbol{q}) . r_{5}(\boldsymbol{q}) \neq 0$ for all admissible state vector $\boldsymbol{q}$.

Proof : We can deduce from the eigenvalues (40) and the right eigenvectors (41) the relation :

$$
\nabla_{\boldsymbol{q}} \lambda_{1}(\boldsymbol{q}) \cdot r_{1}(\boldsymbol{q})=\nabla_{\boldsymbol{q}} \lambda_{5}(\boldsymbol{q}) \cdot r_{5}(\boldsymbol{q})=\hat{a}+\rho \hat{a}^{2} \frac{\partial \hat{a}}{\partial p}
$$

So the proof consists to show that $\hat{a}+\rho \hat{a}^{2} \partial \hat{a} / \partial p$ is always non zero and the principal difficulty is to evaluate the term $\partial \hat{a} / \partial p$. Let us first rewrite, in term of the mass fractions $Y_{k}$, the mixture density (28) as :

$$
\frac{1}{\rho}=\sum_{k=1}^{2} \frac{Y_{k}}{\rho_{k}}
$$

and also the averaged sound speed (29) under the form :

$$
\frac{1}{(\rho \hat{a})^{2}}=\sum_{k=1}^{2} \frac{Y_{k}}{\left(\rho_{k} a_{k}\right)^{2}}
$$

Now let us consider the following differentiation of the sound speed $\hat{a}$ for an arbitrary variable $\phi$ :

$$
\frac{\partial \hat{a}}{\partial \phi}=-\hat{a}\left[\frac{(\rho \hat{a})^{2}}{2} \frac{\partial}{\partial \phi}\left(\frac{1}{(\rho \hat{a})^{2}}\right)-\rho \frac{\partial}{\partial \phi}\left(\frac{1}{\rho}\right)\right]
$$

Using this relation (49) for $\phi=p$ and introducing expressions (47),(48) we get :

$$
\begin{aligned}
& \hat{a}+\rho \hat{a}^{2} \frac{\partial \hat{a}}{\partial p}=\hat{a}-\rho \hat{a}^{3}\left[\frac{(\rho \hat{a})^{2}}{2} \frac{\partial}{\partial p}\left(\sum_{k=1}^{2} \frac{Y_{k}}{\left(\rho_{k} a_{k}\right)^{2}}\right)-\rho \frac{\partial}{\partial p}\left(\sum_{k=1}^{2} \frac{Y_{k}}{\rho_{k}}\right)\right] \\
& \hat{a}+\rho \hat{a}^{2} \frac{\partial \hat{a}}{\partial p}=\hat{a}-\rho \hat{a}^{3}\left[\frac{(\rho \hat{a})^{2}}{2} \sum_{k=1}^{2} Y_{k} \frac{\partial}{\partial p}\left(\frac{1}{\left(\rho_{k} a_{k}\right)^{2}}\right)-\rho \sum_{k=1}^{2} Y_{k} \frac{\partial}{\partial p}\left(\frac{1}{\rho_{k}}\right)\right]
\end{aligned}
$$

Then introducing the coefficients $\psi_{k}=\left(\partial a_{k} / \partial p_{k}\right)_{s_{k}}$ for each phase and recalling that the definition of the phase sound speeds implies $\left(\partial \rho_{k} / \partial p_{k}\right)_{s_{k}}=1 / a_{k}^{2}$, we obtain the following results :

$$
\begin{aligned}
& \hat{a}+\rho \hat{a}^{2} \frac{\partial \hat{a}}{\partial p}=\hat{a}-\rho \hat{a}^{3}\left[-(\rho \hat{a})^{2} \sum_{k=1}^{2} \frac{Y_{k}\left(1+\rho_{k} a_{k} \psi_{k}\right)}{\rho_{k}^{3} a_{k}^{4}}+\rho \sum_{k=1}^{2} \frac{Y_{k}}{\left(\rho_{k} a_{k}\right)^{2}}\right] \\
& \hat{a}+\rho \hat{a}^{2} \frac{\partial \hat{a}}{\partial p}=\hat{a}-\rho \hat{a}^{3}\left[-(\rho \hat{a})^{2} \sum_{k=1}^{2} \frac{Y_{k}\left(1+\rho_{k} a_{k} \psi_{k}\right)}{\rho_{k}^{3} a_{k}^{4}}+\frac{1}{\rho \hat{a}^{2}}\right]
\end{aligned}
$$

which give us after simplifications :

$$
\hat{a}+\rho \hat{a}^{2} \frac{\partial \hat{a}}{\partial p}=\rho^{3} \hat{a}^{5} \sum_{k=1}^{2} \frac{Y_{k}\left(1+\rho_{k} a_{k} \psi_{k}\right)}{\rho_{k}^{3} a_{k}^{4}}
$$

And finally assuming that $\psi_{k}>0$ i.e the phase sound speed increases with the pressure, when the entropy is constant, we get $\left(1+\rho_{k} a_{k} \psi_{k}\right) \neq 0$ and this achieve the proof. 
Remark : We note that if the fluids are governed by the Stiffened-Gas equation of state; the condition $\psi_{k}>0$ is equivalent to $\gamma_{k}>1$ where $\gamma_{k}$ denotes the adiabatic exponent of each phase.

Now let us examine the characteristic field associated to the wave $u$.

Proposition 5 : The characteristic field associated to the wave $\lambda_{2}(\boldsymbol{q})=\lambda_{3}(\boldsymbol{q})=\lambda_{4}(\boldsymbol{q})=u$ is linearly degenerate i.e we have : $\nabla \boldsymbol{q} \lambda_{i}(\boldsymbol{q}) . r_{i}(\boldsymbol{q})=0$ (for $i \in\{2,3,4\}$ ) for all admissible state vector $\boldsymbol{q}$.

Proof: We deduce from the eigenvalues (40) the relation:

$$
\nabla \boldsymbol{q} \lambda_{i}(\boldsymbol{q}) \cdot r_{i}(\boldsymbol{q})=(0,0,1,0,0) \cdot r_{i}(\boldsymbol{q}) \quad \text { for } \quad i \in\{2,3,4\}
$$

Then introducing the right eigenvectors (41) in the relation (53), it is easily checked that $\nabla \boldsymbol{q} \lambda_{i}(\boldsymbol{q}) \cdot r_{i}(\boldsymbol{q})=0$ (for $i \in\{2,3,4\}$ ) and this complete the proof.

\subsection{Riemann's invariants}

In this section we compute the Riemann's invariants of the system. Let us begin with the Riemann's invariants $\omega$ associated to the eigenvalue $\lambda_{1}(\boldsymbol{q})=u-\hat{a}$. The problem is thus to find $\omega$ such as $\nabla \boldsymbol{q} \omega \cdot r_{1}(\boldsymbol{q})=0$. So if we search $\omega$ such that $\nabla \boldsymbol{q} \omega$ is colinear with ${ }^{t} l_{2}(\boldsymbol{q}),{ }^{t} l_{3}(\boldsymbol{q})$ and ${ }^{t} l_{4}(\boldsymbol{q})$, it is easily checked that $s_{1}, s_{2}$ and $Y_{2}$ are three Riemann's invariants associated to this wave. Now if we search $\nabla \boldsymbol{q} \omega$ colinear with ${ }^{t}\left(2 \hat{a} l_{5}\right)=(0,0,1,1 / \rho \hat{a}, 0)$, we get the last Riemann's invariant :

$$
u+\int_{p} \frac{d p}{\rho \hat{a}}
$$

We note that this last expression for $\omega$ is formally equivalent to the well-known expression of the Riemann's invariant $u+2 a /(\gamma-1)$ of the Euler equations of gas dynamics. To summarize, the Riemann's invariants associated to the wave $u-\hat{a}$ are defined by :

$$
\left\{s_{1}, s_{2}, \quad Y_{2}, u+\int_{p} \frac{d p}{\rho \hat{a}}\right\}
$$

After similar algebraic manipulations, it is easily checked that the Riemann's invariants associated to the wave $u+\hat{a}$ are defined by :

$$
\left\{s_{1}, s_{2}, \quad Y_{2}, u-\int_{p} \frac{d p}{\rho \hat{a}}\right\}
$$

On the other hand, the Riemann's invariants associated to the wave $u$ are given by :

$$
\{u, p\}
$$

To close this section, we also note that if the system is written in term of conservative variables, the first four equations of (30) are in conservative form and give us a set of 4 jump conditions that discontinuities have to satisfy :

$$
\left\{\begin{array}{l}
\Delta\left[\alpha_{1} \rho_{1}(u-\sigma)\right]=0 \\
\Delta\left[\alpha_{2} \rho_{2}(u-\sigma)\right]=0 \\
\Delta[\rho u(u-\sigma)+p]=0 \\
\Delta[\rho e(u-\sigma)+p u]=0
\end{array}\right.
$$

with $\Delta \phi=\phi_{R}-\phi_{L}$ and $\sigma$ the velocity of the discontinuity. However, the equation for the volume fraction $\alpha_{2}$ is not in conservative form and therefore, the system must be supplemented or regularized to specify the admissible shock waves. As discussed in [10], such a regularization can be obtained by a close examination of the structure of the large gradient relaxation zones or by a construction of subscale models of the physical processes occuring within the discontinuities. Here, we adopt a simpler approach and rely on artificial viscosity to regularize the model. 


\section{Numerical Approximation}

\subsection{The VFRoe-ncv scheme}

In this section, we describe a quasi-conservative finite volume scheme. The method is based on VFRoe-ncv type scheme [5] i.e on the solution of a linearized Riemann's problem at each interface of the mesh. Consider the following linearized Riemann's problem between the states $(.)_{L}$ and $(.)_{R}$ :

$$
\left\{\begin{array}{l}
\frac{\partial \boldsymbol{q}}{\partial t}+A(<\boldsymbol{q}>) \frac{\partial \boldsymbol{q}}{\partial x}=0 \\
\boldsymbol{q}(x, 0)=\mid \begin{array}{lll}
\boldsymbol{q}_{L} & \text { if } & x<0 \\
\boldsymbol{q}_{R} & \text { if } & x>0
\end{array}
\end{array}\right.
$$

Here, we use the set of variables $\boldsymbol{q}={ }^{t}\left(s_{1}, s_{2}, v_{n}, v_{t}, p, Y_{2}\right)$ where $v_{n}, v_{t}$ are respectively the two components of the vector velocity in the local basis $\left(\boldsymbol{\eta}_{L R}, \boldsymbol{\eta}_{L R}^{\perp}\right)$ where $\boldsymbol{\eta}_{L R}$ is the unit normal vector to the interface. We define $A(<\boldsymbol{q}>)$ by :

$$
A(<\boldsymbol{q}>)=\left(\begin{array}{cccccc}
<v_{n}> & 0 & 0 & 0 & 0 & 0 \\
0 & <v_{n}> & 0 & 0 & 0 & 0 \\
0 & 0 & <v_{n}> & 0 & <1 / \rho> & 0 \\
0 & 0 & 0 & <v_{n}> & 0 & 0 \\
0 & 0 & <\rho \hat{a}^{2}> & 0 & <v_{n}> & 0 \\
0 & 0 & 0 & 0 & 0 & <v_{n}>
\end{array}\right)
$$

where $\langle\rangle=.\left((.)_{L}+(.)_{R}\right) / 2$ denotes the arithmetic average between the states $(.)_{L}$ and $(.)_{R}$. Section 3.1 shows that the matrix $A(<\boldsymbol{q}\rangle)$ is diagonalizable and the solution procedure reduces to the solving of a Riemann's problem for a linear hyperbolic system.

We denote by $r_{i}\left(<\boldsymbol{q}>\right.$ ) (for $i \in\{1, \ldots, 6\}$ ) the right eingenvectors and by $l_{i}(<\boldsymbol{q}>$ ) (for $i \in\{1, \ldots, 6\}$ ) the left eigenvectors of the matrix $A(\langle q\rangle)$, satisfying $\left.{ }^{t} l_{i}(<q\rangle\right) \cdot r_{j}(\langle q\rangle)=\delta_{i j}$. The solution of the problem which depends only to the variable $x / t$ is composed, in the $(x, t)$ plane, of constant states separated by characteristic lines. Here we are interested by the solution at the interface i.e on $x / t=0$.

The approximate state at the interface, noted here $\boldsymbol{q}_{L R}^{*}$, is given by :

$$
\boldsymbol{q}_{L R}^{*}=\boldsymbol{q}\left(\frac{x}{t}=0, \boldsymbol{q}_{L}, \boldsymbol{q}_{R}\right)=\left\{\begin{array}{l}
\boldsymbol{q}_{L}+\sum_{\lambda_{i}<0}{ }^{t} l_{i}(<\boldsymbol{q}>) \cdot\left(\boldsymbol{q}_{R}-\boldsymbol{q}_{L}\right) r_{i}(<\boldsymbol{q}>) \\
\boldsymbol{q}_{R}-\sum_{\lambda_{i}>0}{ }^{t} l_{i}(<\boldsymbol{q}>) .\left(\boldsymbol{q}_{R}-\boldsymbol{q}_{L}\right) r_{i}(<\boldsymbol{q}>)
\end{array}\right.
$$

In the sequel we will denote by $\boldsymbol{Q}={ }^{t}\left(\alpha_{1} \rho_{1}, \alpha_{2} \rho_{2}, \rho u, \rho v, \rho e, \alpha_{2}\right)$ the set of conservative variables where $u, v$ are the two-components of the vector velocity $\boldsymbol{u}$ in the global basis corresponding to the vector $\boldsymbol{q}$ written in entropy variables ${ }^{t}\left(s_{1}, s_{2}, v_{n}, v_{t}, p, Y_{2}\right)$. In particular $\boldsymbol{Q}_{L R}^{*}$ will represent the solution of the linearized Riemann's problem (58) written in conservative variables.

To deal with the non-conservative equation :

$$
\frac{\partial \alpha_{2}}{\partial t}+\boldsymbol{u} . \nabla \alpha_{2}=\alpha_{1} \alpha_{2} \frac{\rho_{1} a_{1}^{2}-\rho_{2} a_{2}^{2}}{\sum_{k=1}^{2} \alpha_{k^{\prime}} \rho_{k} a_{k}^{2}} \operatorname{div} \boldsymbol{u}
$$

we re-write it under the form :

$$
\frac{\partial \alpha_{2}}{\partial t}+\operatorname{div}\left(\alpha_{2} \boldsymbol{u}\right)+B(\boldsymbol{Q}) \operatorname{div} \boldsymbol{u}=0 \quad \text { with } \quad B(\boldsymbol{Q})=\frac{-\alpha_{2} \rho_{1} a_{1}^{2}}{\sum_{k=1}^{2} \alpha_{k^{\prime}} \rho_{k} a_{k}^{2}}
$$

and we write the five equation reduced model as :

$$
\frac{\partial \boldsymbol{Q}}{\partial t}+\operatorname{div} \boldsymbol{F}(\boldsymbol{Q})+\operatorname{div} \boldsymbol{u} \boldsymbol{B}(\boldsymbol{Q})=0
$$


where the vector $\boldsymbol{B}(\boldsymbol{Q})={ }^{t}(0,0,0,0,0, B(\boldsymbol{Q}))$. Integrating this equation on a cell $C_{i}$ gives :

$$
A_{i} \frac{\partial \boldsymbol{Q}_{i}}{\partial t}+\int_{\partial C_{i}} \boldsymbol{F}(\boldsymbol{Q}) \cdot \boldsymbol{n} d l+\int_{C_{i}} \boldsymbol{B}(\boldsymbol{Q}) \operatorname{div} \boldsymbol{u} d \Omega=0 \quad \text { for } \quad i \in\{1, \ldots, N\}
$$

where $N$ is the number of cells and $A_{i}$ the area of the cell $C_{i}$. Then we update variables $\boldsymbol{Q}_{i}$ by the following expression :

$$
A_{i} \frac{\boldsymbol{Q}_{i}^{n+1}-\boldsymbol{Q}_{i}^{n}}{\Delta t}+\sum_{j \in v(i)}\left\|\boldsymbol{n}_{i j}\right\| \boldsymbol{\psi}\left(\boldsymbol{Q}_{i}^{n}, \boldsymbol{Q}_{j}^{n}\right)=0
$$

where $v(i)$ denote the set of cells $C_{j}$ that share an edge with $C_{i}$ and where $\boldsymbol{n}_{i j}=\int_{\partial C_{i j}} \boldsymbol{n} d l$ is the averaged normal vector of the interface $\partial C_{i j}=\partial C_{i} \cap \partial C_{j}$. Defining $\boldsymbol{\eta}_{i j}=\boldsymbol{n}_{i j} /\left\|\boldsymbol{n}_{i j}\right\|$ and

$$
\left\{\begin{array}{l}
\left(v_{n}\right)_{i j}=\boldsymbol{u}_{i j} \cdot \boldsymbol{\eta}_{i j}=u_{i j} \eta_{i j}^{x}+v_{i j} \eta_{i j}^{y} \\
\left(v_{t}\right)_{i j}=\boldsymbol{u}_{i j} \cdot \boldsymbol{\eta}_{i j}^{\perp}=-u_{i j} \eta_{i j}^{y}+v_{i j} \eta_{i j}^{x}
\end{array}\right.
$$

the normal and tangential components of the velocity at the cell interface, we propose the following expression for $\boldsymbol{\psi}\left(\boldsymbol{Q}_{i}^{n}, \boldsymbol{Q}_{j}^{n}\right)$ :

$$
\boldsymbol{\psi}\left(\boldsymbol{Q}_{i}^{n}, \boldsymbol{Q}_{j}^{n}\right)=\boldsymbol{F}\left(\boldsymbol{Q}_{i j}^{*}\right) \cdot \boldsymbol{\eta}_{i j}+\boldsymbol{B}\left(\boldsymbol{Q}_{i}^{n}\right) \boldsymbol{u}_{i j}^{*} \cdot \boldsymbol{\eta}_{i j}
$$

where $\boldsymbol{Q}_{i j}^{*}$ is the solution (60) of the linearized Riemann's problem between the states $(.)_{i}$ and $(.)_{j}$.

The conservative part $\boldsymbol{F}\left(\boldsymbol{Q}_{i j}^{*}\right) \cdot \boldsymbol{\eta}_{i j}$ can be explicitely written :

$$
\boldsymbol{F}\left(\boldsymbol{Q}_{i j}^{*}\right) \cdot \boldsymbol{\eta}_{i j}={ }^{t}\left(\left(\alpha_{1} \rho_{1} v_{n}\right)_{i j}^{*},\left(\alpha_{2} \rho_{2} v_{n}\right)_{i j}^{*},\left(\rho u v_{n}\right)_{i j}^{*}+p_{i j}^{*} \eta_{i j}^{x},\left(\rho v v_{n}\right)_{i j}^{*}+p_{i j}^{*} \eta_{i j}^{y},\left((\rho e+p) v_{n}\right)_{i j}^{*},\left(\alpha_{2} v_{n}\right)_{i j}^{*}\right)
$$

while the non-conservative part $\boldsymbol{B}\left(\boldsymbol{Q}_{i}^{n}\right) \boldsymbol{u}_{i j}^{*} \cdot \boldsymbol{\eta}_{i j}$ is defined by :

$$
\boldsymbol{B}\left(\boldsymbol{Q}_{i}^{n}\right) \boldsymbol{u}_{i j}^{*} \cdot \boldsymbol{\eta}_{i j}={ }^{t}\left(0,0,0,0,0, B\left(\boldsymbol{Q}_{i}^{n}\right)\left(v_{n}\right)_{i j}^{*}\right)
$$

\subsection{Evolution of a contact discontinuity}

As shown in [2], one key point for the simulation of interface problems between compressible fluid is the capability of the scheme to compute an isolated contact discontinuity without pressure oscillations. In the following, we thus study the capability of the scheme to preserve a uniform pressure and velocity flow. Consider an initial flow where pressure and velocity have uniform values $; u_{i-1}=u_{i}=u_{i+1}=u$ and $p_{i-1}=p_{i}=p_{i+1}=p$ but other quantities $\alpha_{k} \rho_{k}, \alpha_{2}$ can be discontinuous. We denote by $\boldsymbol{Q}_{i-1}, \boldsymbol{Q}_{i}, \boldsymbol{Q}_{i+1}$ the conservative variables vectors. Then if we suppose for instance $u>0$, it is easily seen that the solution of the linearized Riemann's problem at each interface is given by :

$$
\left\{\begin{array}{l}
\boldsymbol{Q}_{i-1 / 2}^{*}=\boldsymbol{Q}_{i-1} \\
\boldsymbol{Q}_{i+1 / 2}^{*}=\boldsymbol{Q}_{i} \\
\boldsymbol{Q}_{i+3 / 2}^{*}=\boldsymbol{Q}_{i+1}
\end{array}\right.
$$

And thus the only cell whose value is changed between $t$ and $t+\Delta t$ is the cell downstream of the discontinuity. The value of $\boldsymbol{Q}$ in this cell becomes :

$$
\begin{aligned}
& \boldsymbol{Q}_{i+1}^{n+1}=\boldsymbol{Q}_{i+1}^{n}-\lambda\left(\boldsymbol{F}\left(\boldsymbol{Q}_{i+3 / 2}^{*}\right)-\boldsymbol{F}\left(\boldsymbol{Q}_{i+1 / 2}^{*}\right)\right)-\lambda \boldsymbol{B}\left(\boldsymbol{Q}_{i+1}^{n}\right)\left(u_{i+3 / 2}^{*}-u_{i+1 / 2}^{*}\right) \\
& \boldsymbol{Q}_{i+1}^{n+1}=\boldsymbol{Q}_{i+1}^{n}-\lambda\left(\boldsymbol{F}\left(\boldsymbol{Q}_{i+1}\right)-\boldsymbol{F}\left(\boldsymbol{Q}_{i}\right)\right)-\lambda \boldsymbol{B}\left(\boldsymbol{Q}_{i+1}^{n}\right)\left(u_{i+1}-u_{i}\right) \\
& \boldsymbol{Q}_{i+1}^{n+1}=\boldsymbol{Q}_{i+1}^{n}-\lambda\left(\boldsymbol{F}\left(\boldsymbol{Q}_{i+1}\right)-\boldsymbol{F}\left(\boldsymbol{Q}_{i}\right)\right)
\end{aligned}
$$

with $\lambda=\Delta t / A_{i}$. So the mass conservation equations can be written :

$$
\left(\alpha_{k} \rho_{k}\right)_{i+1}^{n+1}=\left(\alpha_{k} \rho_{k}\right)_{i+1}^{n}-\lambda u\left[\left(\alpha_{k} \rho_{k}\right)_{i+1}^{n}-\left(\alpha_{k} \rho_{k}\right)_{i}^{n}\right] \quad \text { for } \quad k=1,2
$$

And we get for the evolution of the mixture density $\rho=\sum_{k=1}^{2} \alpha_{k} \rho_{k}$ :

$$
\rho_{i+1}^{n+1}=\rho_{i+1}^{n}-\lambda u\left[\rho_{i+1}^{n}-\rho_{i}^{n}\right]
$$


Now if we look at the momentum conservation equation, we have :

$$
\begin{aligned}
& (\rho u)_{i+1}^{n+1}=(\rho u)_{i+1}^{n}-\lambda\left[\rho_{i+1}^{n} u^{2}+p-\rho_{i}^{n} u^{2}+p\right] \\
& (\rho u)_{i+1}^{n+1}=(\rho u)_{i+1}^{n}-\lambda u^{2}\left[\rho_{i+1}^{n}-\rho_{i}^{n}\right]
\end{aligned}
$$

that together with (73) gives:

$$
u_{i+1}^{n+1}=u_{i+1}^{n}=u
$$

Then the total energy equation is written :

$$
(\rho e)_{i+1}^{n+1}=(\rho e)_{i+1}^{n}-\lambda u\left[(\rho e)_{i+1}^{n}-(\rho e)_{i}^{n}\right]
$$

Using the equation for the mixture density (73) and the result $u_{i+1}^{n+1}=u_{i+1}^{n}=u$, we get (where $\varepsilon$ stands for the internal mixture energy) :

$$
(\rho \varepsilon)_{i+1}^{n+1}=(\rho \varepsilon)_{i+1}^{n}-\lambda u\left[(\rho \varepsilon)_{i+1}^{n}-(\rho \varepsilon)_{i}^{n}\right]
$$

Then using the Stiffened-Gas state law for each phase, we have the following expression for the mixture internal energy :

$$
\rho \varepsilon=\sum_{k=1}^{2} \alpha_{k} \frac{p+\gamma_{k} \pi_{k}}{\gamma_{k}-1}
$$

Solving for the pressure with the volume fraction equation :

$$
\left(\alpha_{2}\right)_{i+1}^{n+1}=\left(\alpha_{2}\right)_{i+1}^{n}-\lambda u\left[\left(\alpha_{2}\right)_{i+1}^{n}-\left(\alpha_{2}\right)_{i}^{n}\right]
$$

We get :

$$
p_{i+1}^{n+1}=p_{i+1}^{n}=p
$$

Therefore, this result guarantee that a contact discontinuity will remain at constant velocity and pressure (although it will evolve toward a smeared profile due to the numerical diffusion).

\subsection{Acoustic Solver}

The results of the previous section show that the VFRoe-ncv scheme preserve an isolated contact discontinuity and that the velocity and the pressure must stay constant in this case. Numerical experiment confirm that this is indeed the case except at very low Mach number. This seems surprising because the previous proof is independent of the Mach number. However, this proof assumes that the computations are done with exact arithmetic. In practice, this is not the case and round-off errors perturbate the computations. A close examination of the results shows that the computation of the interface pressure in equation (60) is extremely sensible to round-off errors at small Mach number. This is the reason why we have developped another approximate Riemann solver where the computation of the pressure appear to be less sensible to small random perturbations. This second numerical scheme uses more heavily the mathematical structure of the reduced system. It can be understood as an extension of the acoustic solver described for instance in [17] for the Euler equations of gas dynamics. With respect to the different numerical test, this acoustic solver seems to be very robust with respect to the Mach number and specially for interface problems. The principle of this solver is to write linearized characteristic equations starting on the two side of the discontinuity and to compute their intersection to get the velocity and pressure at the interface. To be more specific we first transform the system of partial differential equations into ordinary differential equations by multiply them with the left eigenvectors :

$$
{ }^{t} l_{i}(\boldsymbol{q}) \cdot\left(\frac{\partial \boldsymbol{q}}{\partial t}+A(\boldsymbol{q}) \frac{\partial \boldsymbol{q}}{\partial x}\right)=0
$$

which can be immediately rewritten :

$$
{ }^{t} l_{i}(\boldsymbol{q}) \cdot\left(\frac{\partial \boldsymbol{q}}{\partial t}+\lambda_{i}(\boldsymbol{q}) \frac{\partial \boldsymbol{q}}{\partial x}\right)=0
$$


Now, denoting $q_{L}^{*}, q_{R}^{*}$, respectively the states on the left and right side of the contact discontinuity and linearizing (82) with respect to $q_{L}$ and $q_{R}$ we get :

$$
\left\{\begin{array}{l}
{ }^{t} l_{1}\left(\boldsymbol{q}_{L}\right) \cdot\left(\boldsymbol{q}_{L}^{*}-\boldsymbol{q}_{L}\right)=0 \\
{ }^{t} l_{5}\left(\boldsymbol{q}_{R}\right) \cdot\left(\boldsymbol{q}_{R}^{*}-\boldsymbol{q}_{R}\right)=0
\end{array}\right.
$$

which gives after some algebraic manipulations :

$$
\left\{\begin{array}{l}
\rho_{L} \hat{a}_{L}\left(u_{L}^{*}-u_{L}\right)+\left(p_{L}^{*}-p_{L}\right)=0 \\
\rho_{R} \hat{a}_{R}\left(u_{R}^{*}-u_{R}\right)-\left(p_{R}^{*}-p_{R}\right)=0
\end{array}\right.
$$

Then using the fact that $u_{L}^{*}=u_{R}^{*}=u^{*}$ and $p_{L}^{*}=p_{R}^{*}=p^{*}$, we get the following expressions for $u^{*}$ and $p^{*}$ :

$$
\left\{\begin{array}{l}
u^{*}=\frac{\rho_{L} \hat{a}_{L} u_{L}+\rho_{R} \hat{a}_{R} u_{R}}{\rho_{L} \hat{a}_{L}+\rho_{R} \hat{a}_{R}}-\frac{p_{R}-p_{L}}{\rho_{L} \hat{a}_{L}+\rho_{R} \hat{a}_{R}} \\
p^{*}=\frac{\rho_{R} \hat{a}_{R} p_{L}+\rho_{L} \hat{a}_{L} p_{R}}{\rho_{L} \hat{a}_{L}+\rho_{R} \hat{a}_{R}}-\frac{\rho_{L} \hat{a}_{L} \rho_{R} \hat{a}_{R}\left(u_{R}-u_{L}\right)}{\rho_{L} \hat{a}_{L}+\rho_{R} \hat{a}_{R}}
\end{array}\right.
$$

And finally the solution of the Riemann's problem is given by :

$$
\boldsymbol{q}\left(\frac{x}{t}, \boldsymbol{q}_{L}, \boldsymbol{q}_{R}\right)=\left\{\begin{array}{lll}
\boldsymbol{q}_{L} & \text { if } & \frac{x}{t}<u_{L}-\hat{a}_{L} \\
\boldsymbol{q}_{L}^{*} & \text { if } u_{L}-\hat{a}_{L}<\frac{x}{t}<u^{*} \\
\boldsymbol{q}_{R}^{*} & \text { if } u^{*}<\frac{x}{t}<u_{R}+\hat{a}_{R} \\
\boldsymbol{q}_{R} & \text { if } u_{R}+\hat{a}_{R}<\frac{x}{t}
\end{array}\right.
$$

The same numerical scheme which has been presented in section 4.1 can be used with this Riemann solver. We also note that as shown in expression (85), in an isolated contact discontinuity with $p_{L}=p_{R}$ and $u_{L}=u_{R}$ the interface values of the velocity and pressure will again have this common value. The results of section (4.2) thus remain valid when this approximate Riemann solver is used. 


\section{$5 \quad$ Numerical results}

\subsection{Multifluid/Interface problems : Validation tests}

\subsubsection{Pure interface advection}

This first validation test is a one dimension interface advection between water and air. The state laws for the air and the water are given by the Stiffened-Gas formulation :

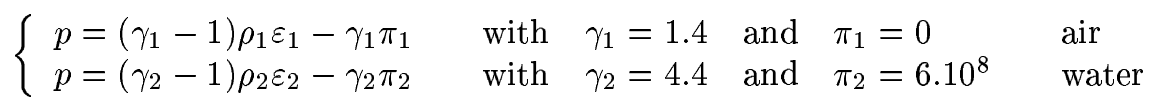

The length of the domain is $1 \mathrm{~m}$ and initally the interface is located at $x=0.5 \mathrm{~m}$. The water with density $\rho_{2}=1000 \mathrm{~kg} \cdot \mathrm{m}^{-3}$ is located on the left side and the air with $\rho_{1}=50 \mathrm{~kg} \cdot \mathrm{m}^{-3}$ is on the right side. Both fluids have same pressure $p=10^{5} \mathrm{~Pa}$ and velocity $u=1000 \mathrm{~m} \cdot \mathrm{s}^{-1}$ at time 0 . A small amount of water (respectively gas) $\alpha_{2}=10^{-8}$ is initially present in the gas (respectively water) side. The discretization is done on 1000 cells grid and the CFL number is equal to 0.6. The results are shown at $229 \mu \mathrm{s}$. In order to compare the results with those given by another similar diffuse interface method, Figure 2 displays the mixture density, the pressure, the velocity and the volume fraction for the present reduced model and also for the "five equation-transport model" of [12] and [3]. For this last model, the numerical method used is the one described in [12]. On this simple test case, the two models are perfectly in agreement and display the same capability to preserve constant velocity and pressure profiles.
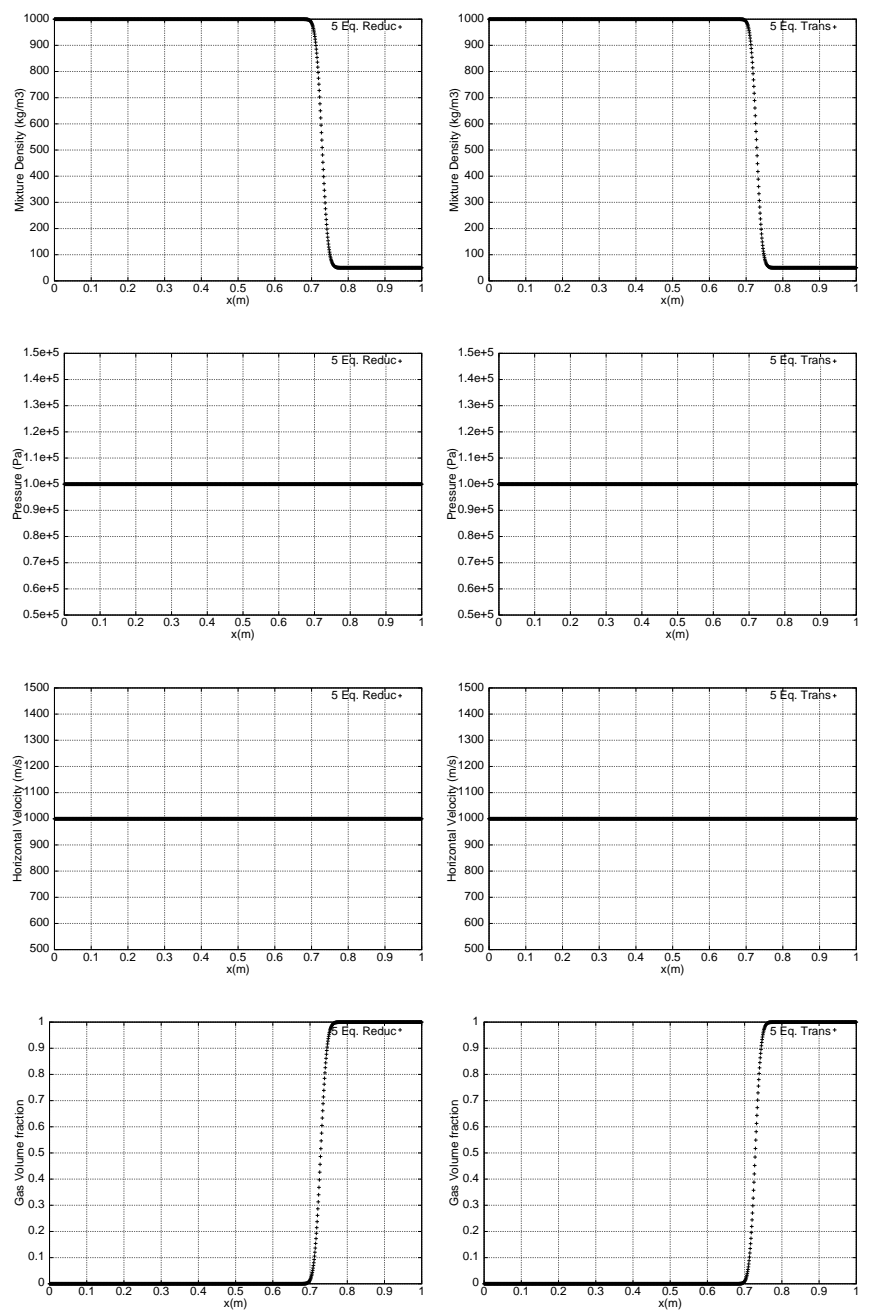

Figure 2: 5 equation reduced model (left) and 5 equation transport model (right) in the pure interface advection : mixture variables. Computed solutions with 1000 cells (symbols). 


\subsubsection{Water-air shock tube}

We consider a shock tube of one meter length filled on the left side $(x<0.7)$ with a high pressure liquid water and on the right side with air. This test problem consists of a classical shock tube with two fluids and admits an exact solution. The state laws are the same than in the previous test case. The initial condition consists in a pressure discontinuity between $p=10^{9} \mathrm{~Pa}$ in the liquid side and $p=10^{5} \mathrm{~Pa}$ in the gas side. As in the previous test case, the right and left chambers contain nearly pure fluids : the volume fraction of the gas in the water chamber is $\alpha_{1}=10^{-8}$ and inversely the water volume fraction is $\alpha_{2}=10^{-8}$ in the gas chamber. Again, we compare the results of the present five equation reduced model with those obtained with the "five equation transport model" of [12] and [3]. Figure 3 displays for the two models the mixture density, the pressure and the velocity. The exact solutions are represented on these curves by a dotted line. This computation uses a mesh with 1000 cells, with a CFL number equal to 0.6 and the results are shown at time $229 \mu$ s. The results for the two models seem to be of comparable accuracy with respect to the exact solution.
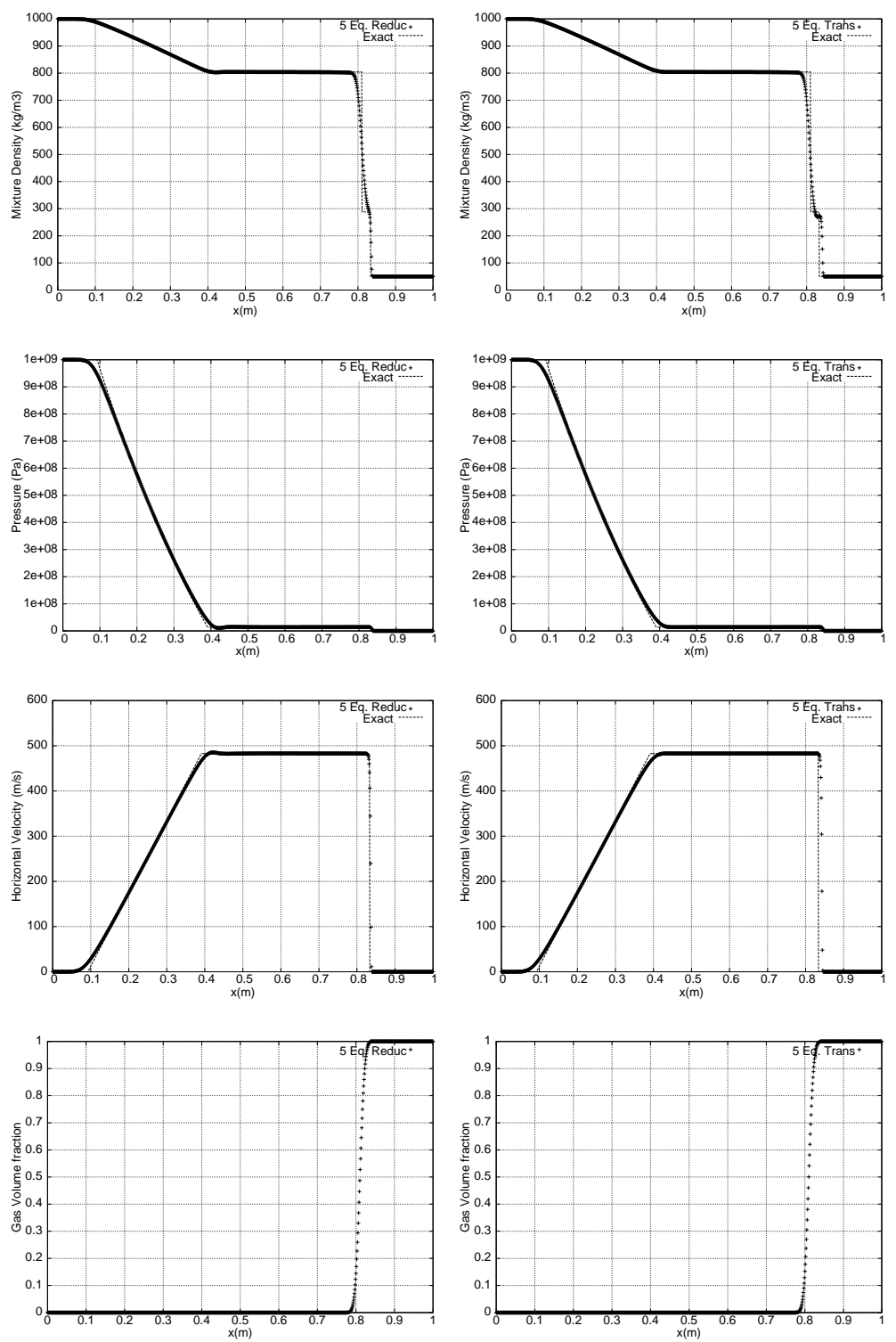

Figure 3: 5 equation reduced model (left) and 5 equation transport model (right) in the water-air shock tube : mixture variables. Computed solutions with 1000 cells (symbols) and exact solutions (dotted lines). 
Although for this problem, these quantities do not have any physical meaning, it can be of interest to look at the phase quantities $\rho_{k}, \varepsilon_{k}$ which respectively stand for the phase densities and internal energies (cf Figure 4). If for the mixture variables the two models have the same behaviour, it is clear that large differences can be noticed on the phase quantities between the two models. Finally let us mention that on this test problem, the results on the phase variables are in good agreement with those published for the seven equation model in [1] or in [11]. In particular, the sharp gradient in the air density and internal energy located upstream of the contact discontinuity are also present in the results obtained with the seven equation model. (Compare Figure 4 (left) with for instance Figure 7 of [1])
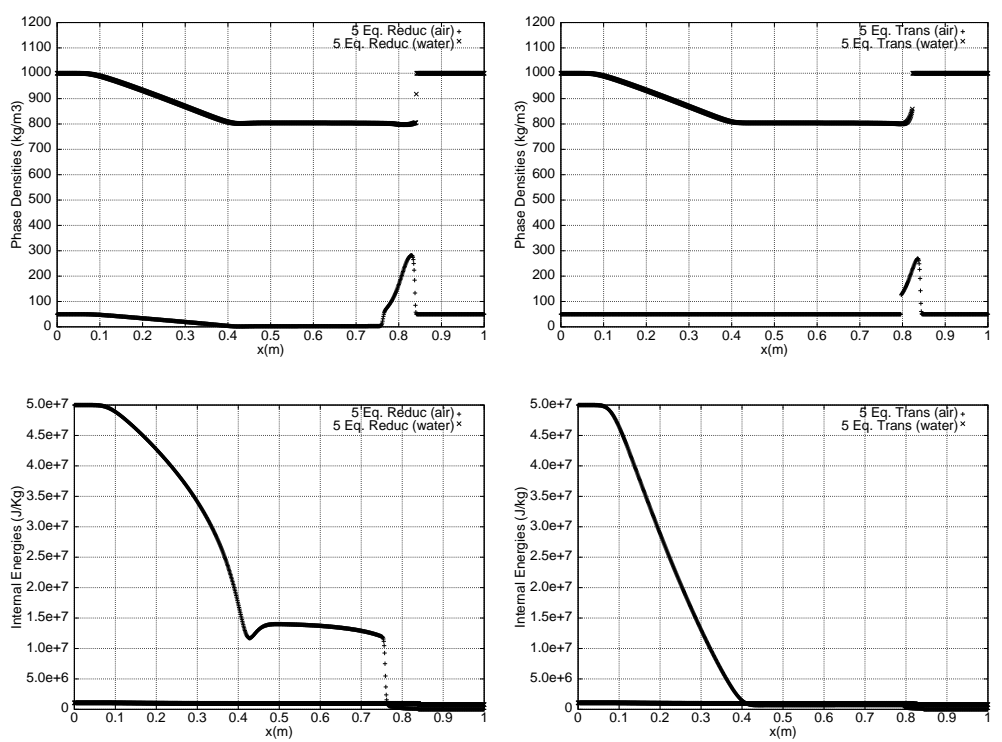

Figure 4: 5 equation reduced model (left) and 5 equation transport model (right) in the water-air shock tube : phase variables. Computed solutions with 1000 cells (symbols). 


\subsection{Two-phase flow problems : Comparison with the seven equation Model}

\subsubsection{First two-phase flow problem}

Our second series of numerical experiments deals with two-phase flow and consider problems where the two phases are simultaneously present at the same location. The first experiment considers the same problem than in section 5.1.2 except that the volume fraction is constant and equal to $\alpha_{1}=0.5$ everywhere in the domain. On the left side $(x<0.5)$ the pressure is $10^{9} \mathrm{~Pa}$ while it is equal to $10^{5} \mathrm{~Pa}$ on the right side. The velocity is zero at time 0. Again the discretization is done on a 1000 cells grid and the CFL number is fixed and equal to 0.6. The results are shown at time $200 \mu \mathrm{s}$. We compare in Figure 5 these results with those obtained by the seven equation model of [1]. The numerical method used to solve the seven equation model is the one described in [1], except that the relaxation procedures have been improved as described in [11]. The results are in perfect agreement and this confirms that the present five equation model is a correct asymptotic limit of the seven equation model in the limit of zero relaxation time. In particular, we observe that even if the initial composition of the mixture is constant, it evolves in space and time and that this evolution is the same in the results obtained with the two models.
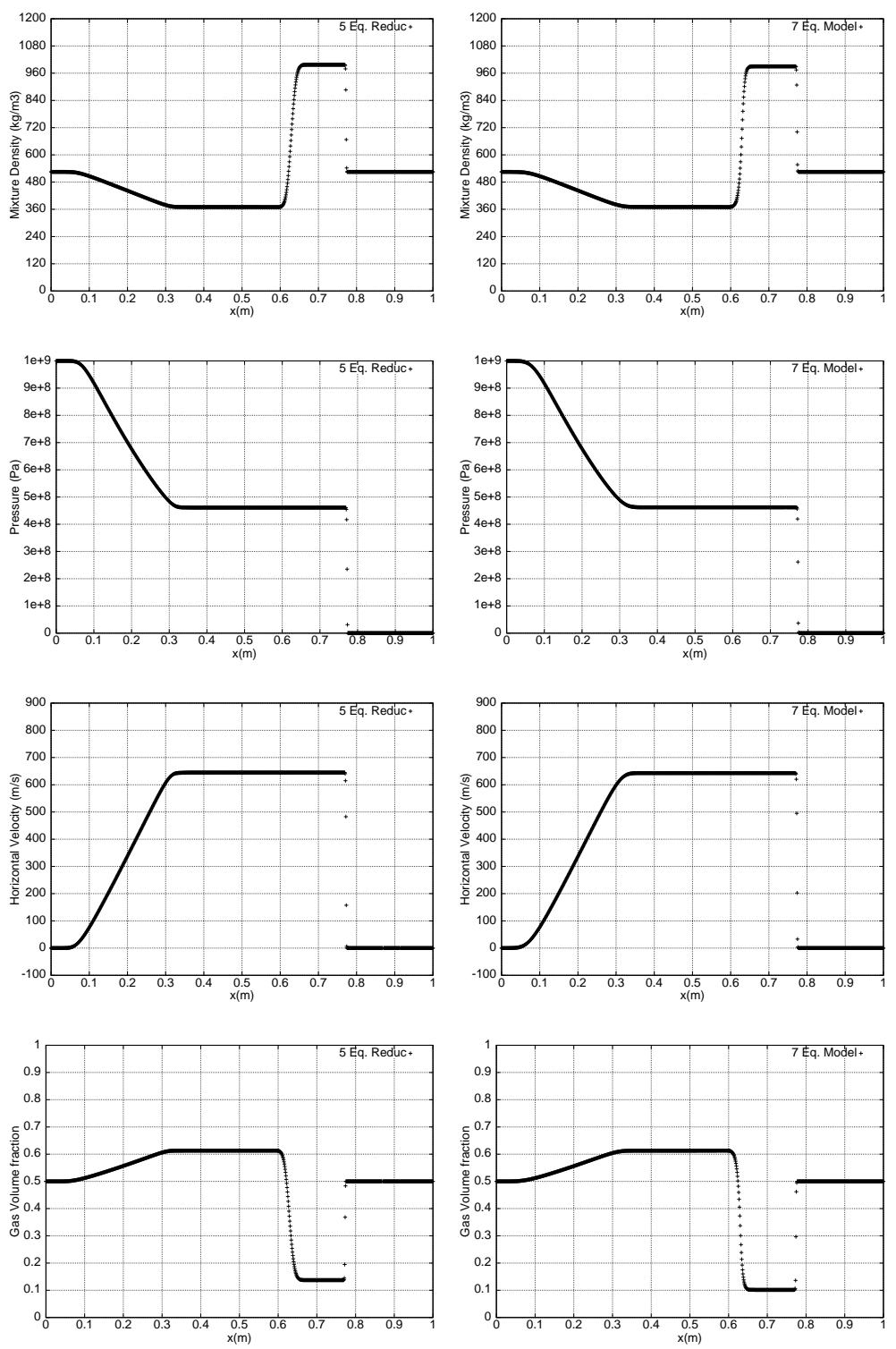

Figure 5: 5 equation reduced model (left) and 7 equation model (right) in the first two-phase flow problem. Computed solutions with 1000 cells (symbols). 


\subsubsection{Second two-phase flow problem}

The same problem than previously is now considered except that we allow a change in the composition of the mixture. We also change the initial density of the gas for $\rho_{1}=1 \mathrm{~kg} \cdot \mathrm{m}^{-3}$. The initial conditions are thus $\rho_{1}=1 \mathrm{~kg} \cdot \mathrm{m}^{-3}, \rho_{2}=1000 \mathrm{~kg} \cdot \mathrm{m}^{-3}, u=0 \mathrm{~m} \cdot \mathrm{s}^{-1}$ everywhere in the domain while :

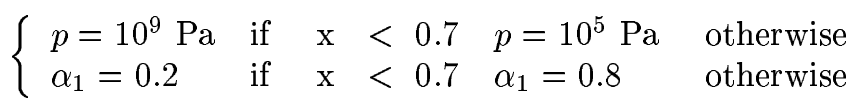

The results are shown at time $200 \mu \mathrm{s}$ (cf Figure 6). The pressure and velocity curves computed with the two models are identical but we notice some differences between the results obtained with the two models in the volume fraction and mixture density profiles. In particular, the post-shock values of the mixture density and volume fraction are not the same and the seven equation model shows an oscillation near the contact discontinuity zone. At the present time, these differences remains unexplainable. Further experiments done by changing the relaxation procedures or the hyperbolic solver in the numerical solution of the seven equation model do not change the results and therefore, it seems that these features are not numerical artifacts but are indeed present in the solution of the seven equation model.
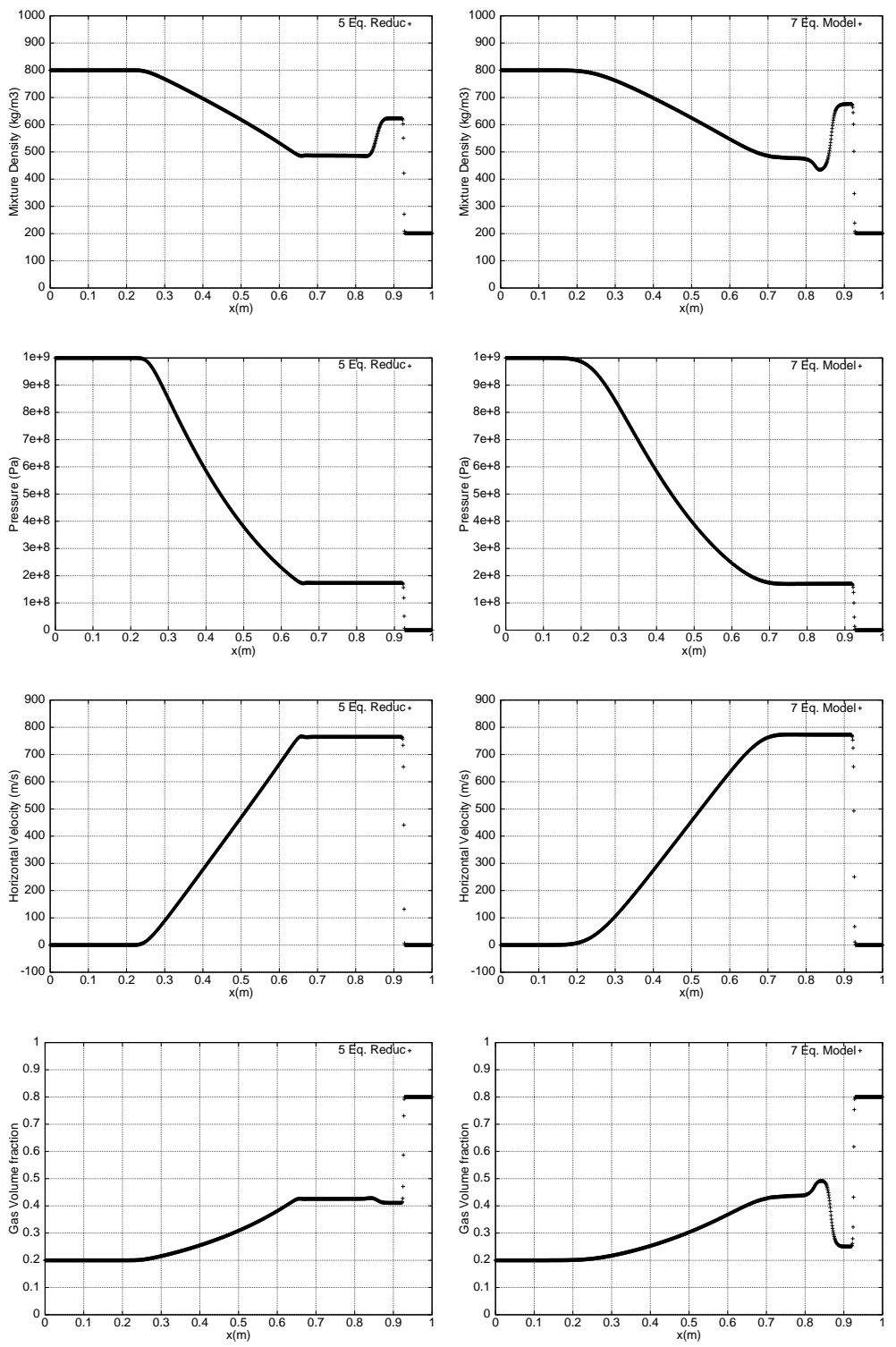

Figure 6: 5 equation reduced model (left) and 7 equation model (right) in the second two-phase flow problem. Computed solutions with 1000 cells (symbols). 


\subsubsection{Shock propagation in solid alloys}

We now evaluate the model capabilities for the computation of shock waves in a two-phase mixture for a test problem involving strong shocks. This type of experiment is particularly important if one want to use the model in DDT studies. Althoug the model does not admit a full set of Rankine-Hugoniot relations it is possible to solve the equations in an unsteady regime and to determine a numerical velocity of the shock. This will be done in simulating an impact situations in a two-phase mixtures as described in Figure 7.

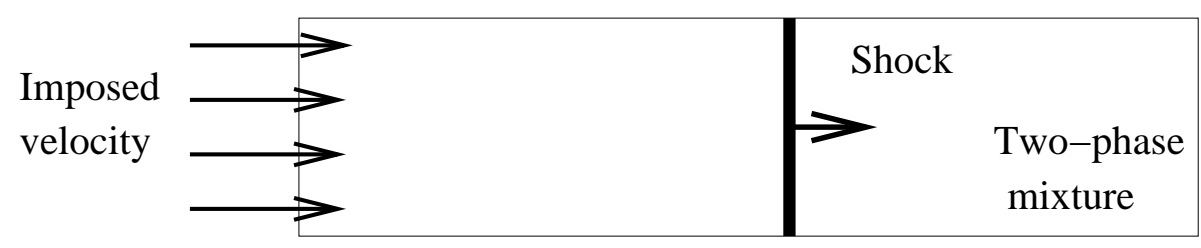

Figure 7: Schematic representation of the two-phase impact problem

In order to evaluate the computed results, we will compare them with those obtained with the seven equation model and with experimental data. Actually, for many material, the relation between the shock velocity and the impact velocity is a linear relation, intrinsically characteristic of the material and experimentally determined :

$$
u_{s}=a_{0}+s u_{p}
$$

where $a_{0}$ is the material sound speed under atmospheric conditions, $u_{s}$ the shock velocity, $u_{p}$ the impact velocity and $s$ is a dimensionless constant. The relation (88) is experimentally available for some alloys. Here, we will consider an epoxy/spinel alloy. The equation of state of the two phases are respectively choosen as :

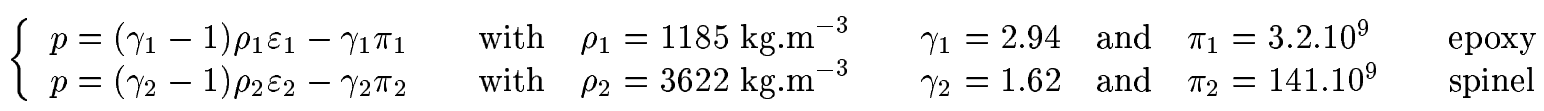

By computing several unsteady problems with variable impact velocity, we will be able to numerically determine the curve (88) and to compare it with the experimental data. Figure 8 diplays the results at different time $=30,60$ and $90 \mu \mathrm{s}$ in a computation where the impact velocity is $u_{p}=3000 \mathrm{~m} / \mathrm{s}$ and the proportion of epoxy is $\alpha_{1}=0.595$. Several computations of this type give the results shown in Figure 9. All the computations use a 1000 cells grid and the CFL number is equal to 0.6. We notice a very good agreement with the experimental data. In addition, we also show in Figure 9 the results obtained with the seven equation model. We note that the present results are of comparable accuracy with those obtained by this model. 

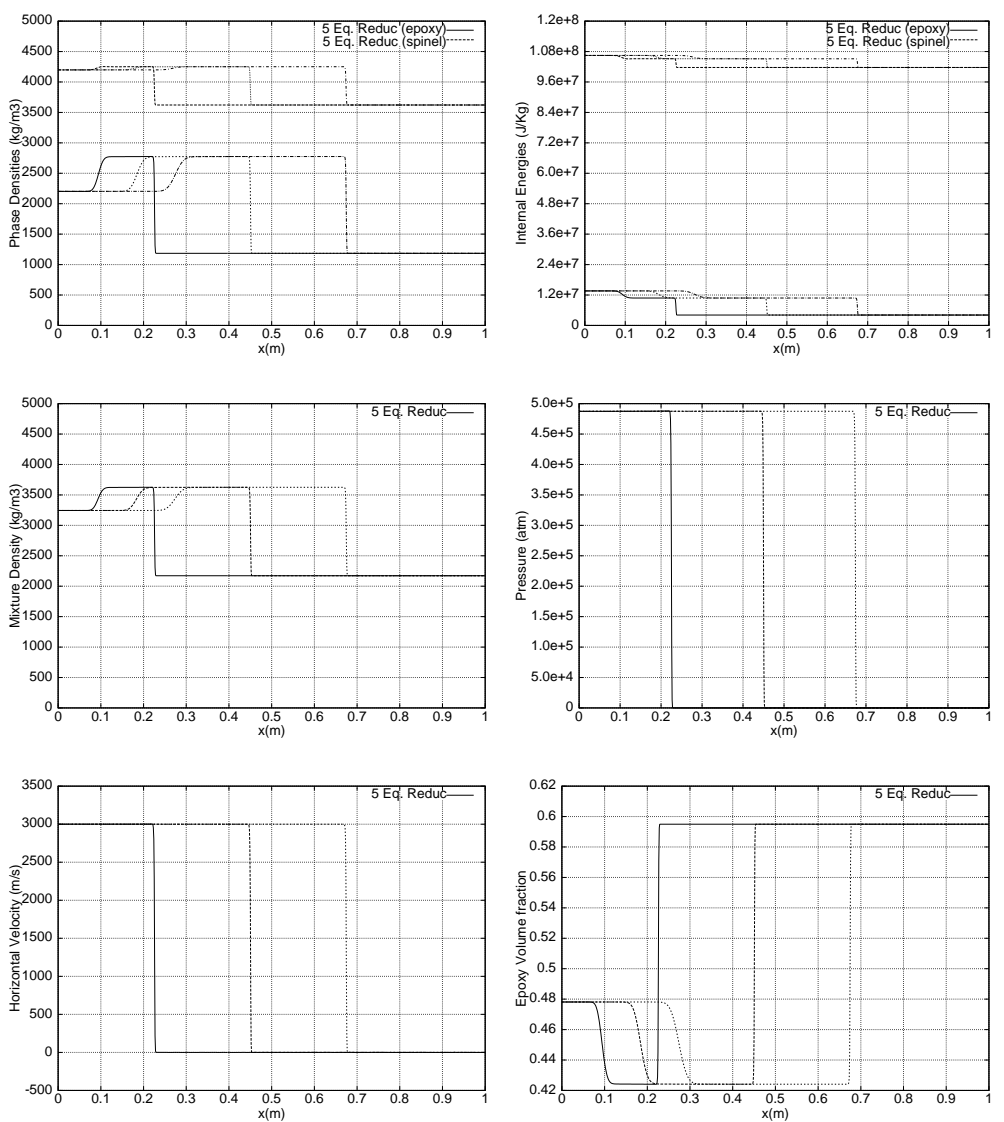

Figure 8: Results of the 5 equation reduced model for the epoxy/spinel under shock impact at $3000 \mathrm{~m} / \mathrm{s}$. Computed solution with 1000 cells.

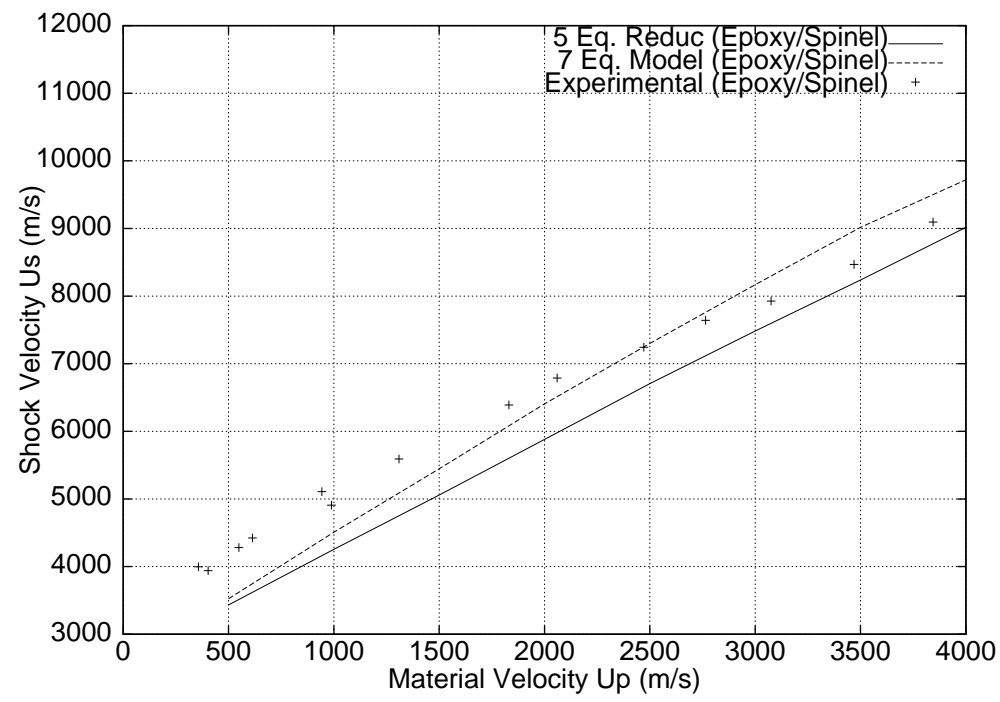

Figure 9: 5 equation reduced model (solid line) and 7 equation model (dotted line) in the Mixture Hugoniot (epoxy/spinel) problem and experimental data (symbols). 


\subsection{Two dimensional tests}

We end these series of numerical experiments by some relevant two-dimensional test-cases. These last experiments are computed with a second order MUSCL technique for the space discretization. The time scheme uses the second order, three stage TVD Runge-Kutta discretization described in [15].

\subsubsection{First two dimensional test : Bubble drop}

This first test shows the drop of a heavy bubble under the effect of the gravity in a closed box as described in Figure 10. Although it seems simple, this computation presents several numerical difficulties. In particular, the Mach number in this computation is extremely low (it is equal to zero at time $t=0$ and increases slighly up to a value of $10^{-2}$ in the course of the computation). This is for this test-case that we had to build the acoustic Riemann solver described in section 4.3. The box is one meter large and two meters high and the mesh is composed of $50 \times 100$ points. In this computation, the CFL number was equal to 0.8. Figure 11 shows the isovalues of the volume fraction at different times. Although an accurate simulation of this problem would require a finer mesh (or an adaptive procedure to follow the interface) the results are very promising. In particular, the numerical diffusion do not prevent the developpement of interface instabilities and the volume fraction remains bounded.

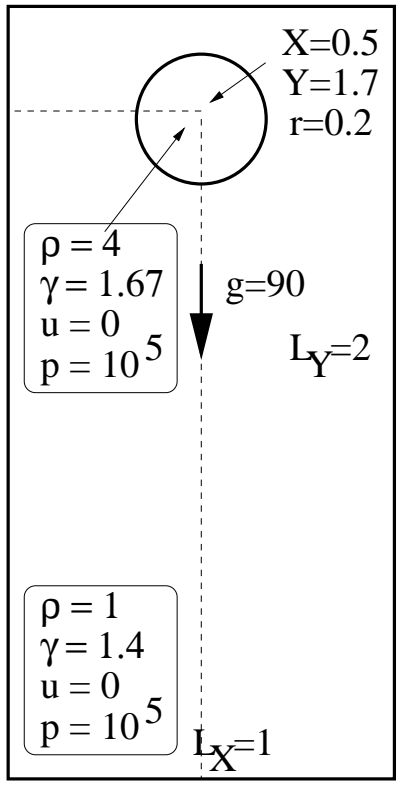

Figure 10: Initial configuration for the first two dimensional test : bubble drop. 

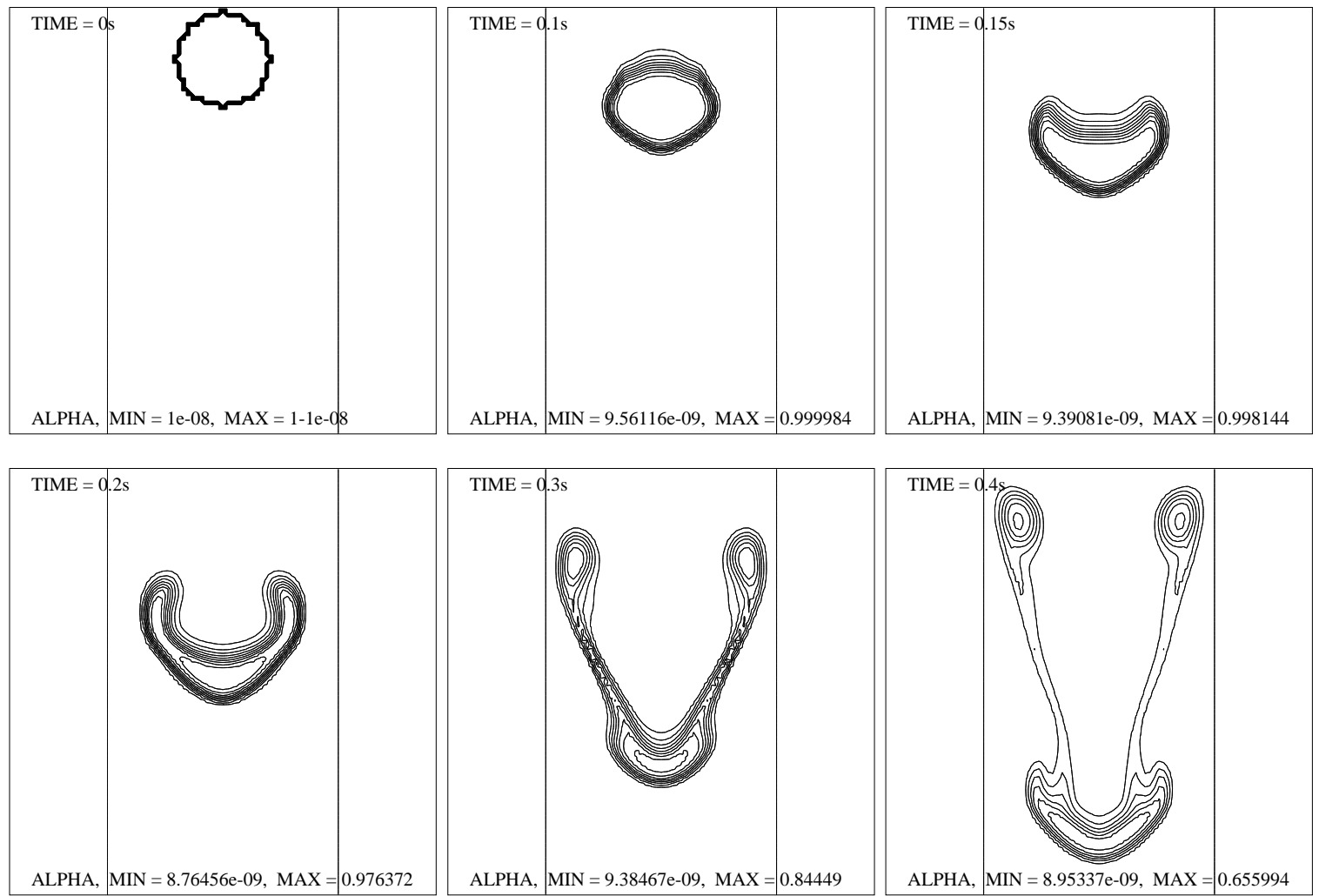

Figure 11: Isovalues of the volume fraction for the first two dimensional test : bubble drop.

\subsubsection{Second two dimensional test}

The second test-case is an idealized representation of the sudden heating of a sphere of light material enclosed in a shell of dense one. This test case has been initially considered in [12]. On Figure 12, we present the initial conditions of the problem. The mesh contains $400 \times 400$ nodes and the CFL number is equal to 0.8 . The mesh is regular. Due to the discretization of the circular interfaces, in addition to the development of shock waves in the direction normal to the interfaces, hydrodynamical instabilities of Richtmyer-Meshkov type will develop. Figure 13 shows the isovalues of the volume fraction at time $=0,1.58 \mathrm{~ms}, 2.63 \mathrm{~ms}$. We clearly observe the developement of these instabilities, producing an intense mixing of the heavy and light fluid near the interface. We also note that although the present results are not totally identical with those obtained in [12], a good agreement with these results is reached.

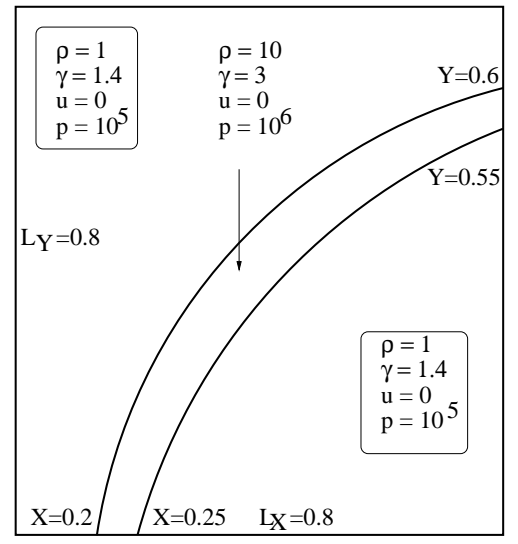

Figure 12: Initial configuration for the second two dimensional test. 

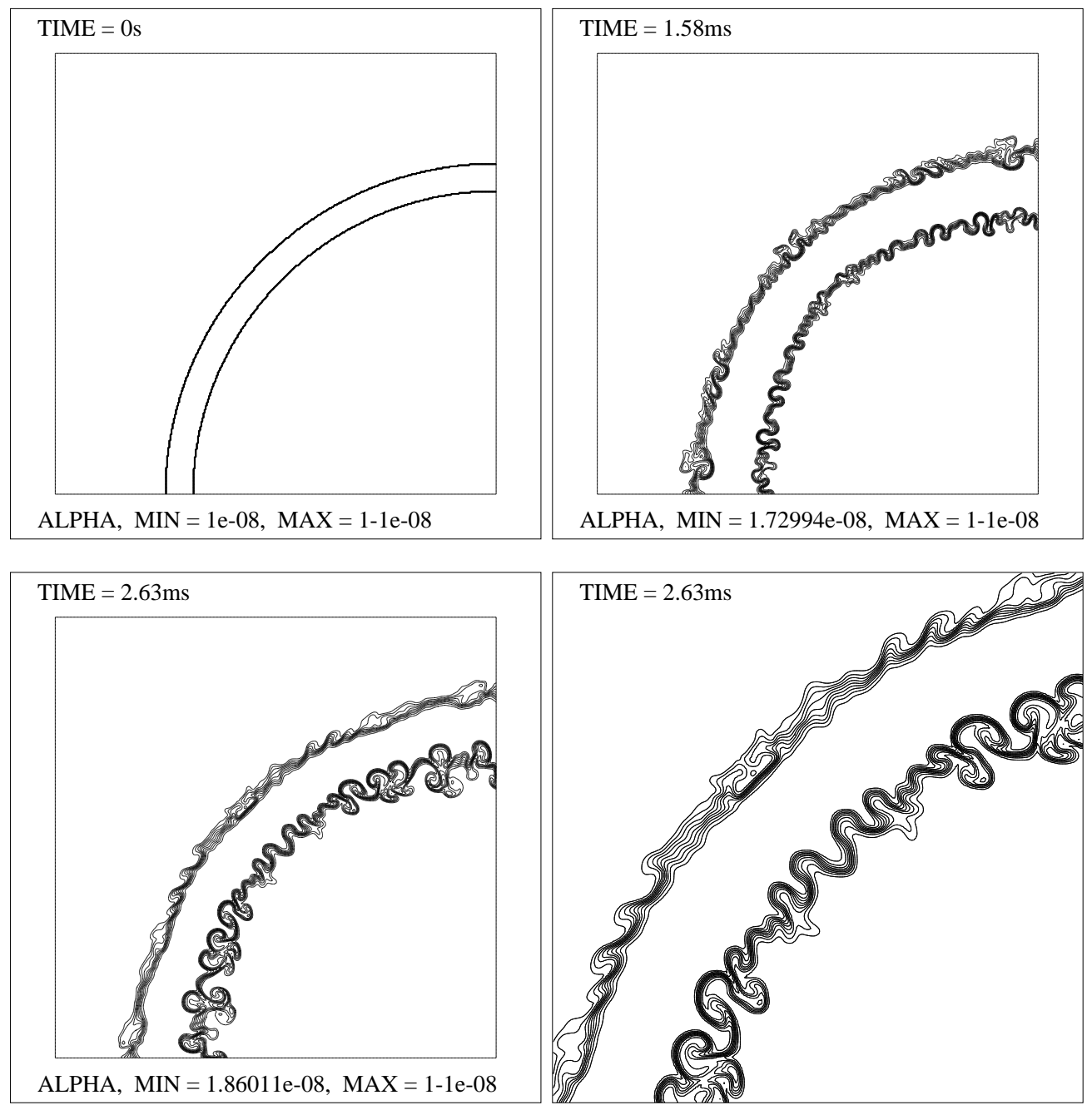

Figure 13: Isovalues of the volume fraction for the second two dimensional test. 


\section{Conclusion}

We have derived a five equation reduced model from an asymptotic analysis in the limit of zero relaxation time of a seven equation two velocity, two pressure model. Although, this model cannot be cast in conservative form, the mathematical structure of the model have been analyzed and shown to be very close to the structure of the Euler equations of fluid dynamics. This model presents an interesting alternative to the use of the seven equation model : it is cheaper, simpler to implement and is easily extensible to an arbitrary number of materials. For instance, in three dimensions, for a number $k$ of different material, the two velocity, two pressure model uses $6 k-1$ variables while the reduced model will use only $2 k+3$ variables.

From a numerical point of view, we have proposed two different approximation schemes of this system. The first one (VFRoe-ncv) relies on an approximate linearized Riemann solver. The second one, that is useful for the simulation of interface problems in low Mach number flows, uses the mathematical structure of the model and relies on the linearization of characteristic relations.

The numerical results show that the reduced five equation model is able of accurate computations of interface problems between compressible material as well as of some two-phase flow problems where pressure and velocity equilibrium between the phases is reached. 


\section{References}

[1] R. Abgrall and R. Saurel. A Multiphase Godunov Method for Compressible Multifluid and Multiphase Flows. Journal of Computational Physics, 150:425-467, 1999.

[2] R. Abgrall and R. Saurel. A simple method for compressible multifluid flows. SIAM J. Sci. Comput., 21 (3):1115-1145, 1999.

[3] G. Allaire, S. Clerc, and S. Kokh. A Five-Equation Model for the Simulation of Interfaces between Compressible Fluids. Journal of Computational Physics, 181:577-616, 2002.

[4] M.R. Baer and J.W. Nunziato. A two-phase mixture theory for the deflagration-to-detonation transition (DDT) in reactive granular materials. Journal of Multiphase Flows, 12:861-889, 1986.

[5] T. Buffard, T. Galouët, and J.M. Hérard. A sequel to a Rough Godunov Scheme : Application to Real Gases. Computers and Fluids, 29:673-709, 2000.

[6] F. Coquel, J.M. Hérard, and N. Seguin. Closure laws for a two-fluid two-pressure model. C. R. Acad. Sci. Paris, Ser. I 334:1-6, 2002.

[7] D.A. Drew and S.L. Passman. Theory of Multicomponent Fluids., volume 135 of APPLIED MATHEMATICAL SCIENCES. Springer, New York, 1998.

[8] E. Godlewski and P.A. Raviart. Numerical Approximation for Hyperbolic systems of Conservation Laws. Spinger-Verlag, 1996.

[9] M. Ishii. Thermo-Fluid Dynamic Theory of Two-Phase Flow. Direction des études et recherches d'électricité de France. Eyrolles, 1975.

[10] A.K. Kapila, J.B. Bdzil, R. Menikoff, S.F. Son, and D.S. Stewart. Two-phase modelling of DDT in granular materials : reduced equations. Physics of Fluids, 13:3002-3024, 2001.

[11] MH. Lallemand and R. Saurel. Pressure relaxation procedures for multiphase compressible flows. Technical Report 4038, INRIA, 2000.

[12] J. Massoni, R. Saurel, B. Nkonga, and R. Abgrall. Propositions de méthodes et modèles Eulériens pour les problèmes à interfaces entre fluides compressibles en présence de transfert de chaleur. International Journal of Heat and Mass Transfer, 45 No 6:1287-1307, 2001.

[13] R. Natalini. Recent Mathematical Results on Hyperbolic Relaxation Problems. Analysis of systems of conservation laws, (Aachen, 1997), Chapman and Hall/CRC, Boca Raton, FL, pages 128-198, 1999.

[14] V.H. Ransom and D.L Hicks. Hyperbolic Two-Pressure Models for Two Phase Flow. Journal of Computational Physics, 53:124-151, 1984.

[15] C.W Shu and S. Osher. Efficient Implementation of Essential Non oscillatory Shock capturing schemes. Journal of Computational Physics, 77:439-471, 1998.

[16] H.B. Stewart and B. Wendroff. Two-phase flow : Models and methods. Journal of Computational Physics, $56: 363-409,1984$.

[17] E.F. Toro. Riemann Solvers and numerical methods for fluid dynamics. Springer Verlag, Berlin, 1997. 


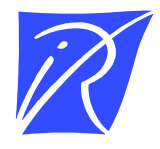

Unité de recherche INRIA Sophia Antipolis 2004, route des Lucioles - BP 93 - 06902 Sophia Antipolis Cedex (France)

Unité de recherche INRIA Lorraine : LORIA, Technopôle de Nancy-Brabois - Campus scientifique 615, rue du Jardin Botanique - BP 101 - 54602 Villers-lès-Nancy Cedex (France)

Unité de recherche INRIA Rennes : IRISA, Campus universitaire de Beaulieu - 35042 Rennes Cedex (France)

Unité de recherche INRIA Rhône-Alpes : 655, avenue de l'Europe - 38330 Montbonnot-St-Martin (France)

Unité de recherche INRIA Rocquencourt : Domaine de Voluceau - Rocquencourt - BP 105 - 78153 Le Chesnay Cedex (France)

Éditeur

INRIA - Domaine de Voluceau - Rocquencourt, BP 105 - 78153 Le Chesnay Cedex (France)

http://www.inria.fr

ISSN 0249-6399 